ARTICLE

\title{
Disease-associated gut microbiome and metabolome changes in patients with chronic obstructive pulmonary disease
}

Kate L. Bowerman (10 1,5, Saima Firdous Rehman (1) 2,5, Annalicia Vaughan ${ }^{3}$, Nancy Lachner ${ }^{1}$, Kurtis F. Budden², Richard Y. Kim4, David L. A. Wood', Shaan L. Gellatly², Shakti D. Shukla (1) ${ }^{2}$, Lisa G. Wood², Ian A. Yang ${ }^{3}$, Peter A. Wark ${ }^{2,6}$, Philip Hugenholtz (1) ${ }^{1,6} \&$ Philip M. Hansbro (iD) 2,4,6凶

Chronic obstructive pulmonary disease (COPD) is the third commonest cause of death globally, and manifests as a progressive inflammatory lung disease with no curative treatment. The lung microbiome contributes to COPD progression, but the function of the gut microbiome remains unclear. Here we examine the faecal microbiome and metabolome of COPD patients and healthy controls, finding 146 bacterial species differing between the two groups. Several species, including Streptococcus sp000187445, Streptococcus vestibularis and multiple members of the family Lachnospiraceae, also correlate with reduced lung function. Untargeted metabolomics identifies a COPD signature comprising 46\% lipid, 20\% xenobiotic and $20 \%$ amino acid related metabolites. Furthermore, we describe a disease-associated network connecting Streptococcus parasanguinis_B with COPD-associated metabolites, including $\mathrm{N}$-acetylglutamate and its analogue $\mathrm{N}$-carbamoylglutamate. While correlative, our results suggest that the faecal microbiome and metabolome of COPD patients are distinct from those of healthy individuals, and may thus aid in the search for biomarkers for COPD.

\footnotetext{
${ }^{1}$ Australian Centre for Ecogenomics, School of Chemistry and Molecular Biosciences, The University of Queensland, Brisbane, QLD, Australia. ${ }^{2}$ Priority Research Centre for Healthy Lungs, Hunter Medical Research Institute, and The University of Newcastle, Newcastle, NSW, Australia. ${ }^{3}$ Thoracic Research Centre, Faculty of Medicine, The University of Queensland, and Department of Thoracic Medicine, The Prince Charles Hospital, Brisbane, QLD, Australia. ${ }^{4}$ Centre for Inflammation, Centenary Institute \& University of Technology Sydney, School of Life Sciences, Faculty of Science, Sydney, NSW, Australia. ${ }^{5}$ These authors contributed equally: Kate L. Bowerman, Saima Firdous Rehman. ${ }^{6}$ These authors jointly supervised this work: Peter A. Wark, Philip Hugenholtz, Philip M. Hansbro. ${ }^{凶}$ email: philip.hansbro@uts.edu.au
} 
C hronic obstructive pulmonary disease (COPD) is a heterogeneous disease with pulmonary pathologies, including chronic bronchitis, airway remodelling and emphysema that impair lung function. It has numerous systemic comorbidities such as cardiovascular disease, colitis and osteoporosis ${ }^{1,2}$. It is the third leading cause of death globally ${ }^{3}$, with the primary risk factor being the inhalation of cigarette smoke, air pollution or other noxious particles ${ }^{4,5}$. However, reportedly only $20-25 \%$ of smokers develop $\mathrm{COPD}^{6}$, and while some genetic risk factors have been described ${ }^{4}$, other factors such as inflammatory and immune responses are important in pathogenesis ${ }^{7}$.

Current approaches to COPD therapy are limited and aim to manage symptoms and reduce exacerbations. High-dose-inhaled corticosteroids are widely employed, but their efficacy is limited to reducing exacerbation frequency or, combined with bronchodilators, improving COPD symptoms ${ }^{8}$. Many patients do not respond to steroid treatment ${ }^{7}$, and these therapies fail to modify the factors that initiate and drive disease progression, do not reverse tissue lesions or improve mortality and predispose to serious respiratory infection and pneumonia ${ }^{8,9}$.

COPD is punctuated by exacerbations that worsen symptoms. Viruses and bacteria in the respiratory tract are associated with disease exacerbation; however, the heterogeneity of the disease and difficulties in sampling the lung make the exact nature of the relationship difficult to interpret ${ }^{10,11}$. Recently, the respiratory tract microbiome has emerged as a contributing factor in COPD progression outside of exacerbations with substantial overlap in identified viruses and bacteria during stable and exacerbated disease ${ }^{12}$. Comparison of sputum and bronchoalveolar lavage fluid (BALF) between stable COPD patients and healthy controls identified an increased abundance of Moraxella, Streptococcus, Veillonella, Eubacterium and Prevotella in disease ${ }^{13,14}$. However, other studies of BALF reported increased Prevotella enoeca but no difference in Streptococcus ${ }^{15}$. Comparisons of lung explants identified increased Proteobacteria and reduced Firmicutes and Bacteroidetes with decreased abundance of Streptococcus, Haemophilus influenza and Prevotella spp. in COPD ${ }^{16}$. Reduced bacterial diversity occurs in stable COPD patient sputum compared to healthy controls ${ }^{13}$; however, both increased and consistent diversity has been reported in BALF ${ }^{14,15}$. These studies suggest that the lung microbiome does not reproducibly change in COPD, which may be related to its transient nature produced by the balancing forces of immigration and elimination that typically impede long-term colonisation ${ }^{17,18}$.

The co-morbidity of colitis suggests that the 'gut-lung axis' may be important in COPD pathogenesis ${ }^{19}$. Thus, we hypothesised that changes in the permanently colonised gut environment may contribute to pathogenesis and be a more reliable indicator of COPD. The concept of the gut-lung axis, describing the common mucosal immune system of the lung and gastrointestinal tract, implicates roles for the gut microbiome in regulating inflammation in acute and chronic respiratory disease including $\mathrm{COPD}^{18,19}$. Several studies implicate disturbances in the abundance or metabolism of gut bacteria in asthma and allergic airway disease ${ }^{20-22}$. In addition, the gut microbiome regulates host immune responses to respiratory infection ${ }^{19,23}$, and may, therefore, contribute to exacerbation frequency in COPD. COPD patients have increased incidence of gastrointestinal disturbances such as ulcerative colitis and Crohn's disease and vice versa ${ }^{24,25}$, indicating potential roles for the gut microbiome in the disease. However, the gastrointestinal microbiome of COPD patients has not been assessed 26,27 .

Here we compare the composition and functional potential of the gut microbiome in COPD patients with those of healthy controls, using untargeted faecal metagenomics and metabolomics. We describe an altered gut microbiome and metabolome associated with the disease. Several strepotococci and members of the family Lachnospiraceae discriminate between COPD patients and healthy controls in addition to correlating with impaired lung function. The metabolomic analysis identifies a shortlist of metabolites that may be potential biomarkers for validation in future studies. These findings support the gut microbiome and metabolome as being altered in association with COPD and highlight the need for further exploration of this environment to uncover whether it plays an active role in disease progression via the gut-lung axis.

\section{Results}

Participant profiles. We separately characterised the gut microbiome and metabolic profiles in COPD by analysing stool from individuals satisfying the global initiative for chronic obstructive lung disease (GOLD) criteria and healthy controls. A total of 28 COPD patients $(54 \%$ female) and 29 healthy controls $(66 \%$ female) were assessed, all during periods of stable disease (Supplementary Data 1). Information on GOLD status, dietary habits, smoking status and medication history was collected, along with spirometry and blood cell counts (Supplementary Data 1-5). COPD patients include four classified as GOLD I, 11 as GOLD II, eight as GOLD III and 11 as GOLD IV. The COPD cohort was older than healthy controls (mean age of 67 vs. $60, p=0.012$ ) and had a significantly higher proportion of past smokers $(p=0.005)$. Daily fibre intake was lower in COPD patients, while pulse rate, total white blood cell, neutrophil, monocyte and eosinophil counts were significantly higher. No significant differences were observed in body mass index (BMI), systolic or diastolic blood pressure, the proportion of current smokers, daily energy, carbohydrate, fat, protein, sugar or starch intake, haemoglobin, total red blood cell, platelet, lymphocyte or basophil counts between the groups (Supplementary Data 1).

Faecal microbiome taxonomic indicators of COPD using $16 \mathrm{~S}$ rRNA gene sequencing. To compare the gut bacterial community composition between COPD patients and healthy individuals, we initially undertook $16 \mathrm{~S}$ rRNA gene sequencing. In total, 4285 sequence variants were identified across all 57 faecal samples. After filtering for sequence variants present in at least two samples with a minimum relative abundance of $0.05 \%$, 977 sequence variants were retained for community analysis. A significant difference in overall community composition was observed between COPD and healthy gut microbiomes (Fig. 1a, $p<0.0001$ PERMANOVA of Bray-Curtis distances), without a significantly altered level of diversity $\left(p_{\text {Shannon }}=0.329\right.$, $\left.p_{\text {SimpsonInverse }}=0.291\right)$. COPD status explained $4 \%$ of the between-sample variability indicating substantial inter-individual differences that remained largely uncaptured by the addition of further demographic variables (Supplementary Data 6). Sequence variants contributing to the distinction between the groups were identified using multivariate sparse partial least-squares discriminant analysis (sPLS-DA, Fig. 1b-d, Supplementary Data 7). Genera increased in abundance in COPD include Streptococcus and Rothia, both common oral bacteria as well as occurring in the gut $^{28}$, Romboutsia and Intestinibacter from the family Peptostreptococcaceae and Escherichia. Genera decreased in COPD include Bacteroides, Roseburia and Lachnospira from the family Lachnospiraceae and several unnamed genera of Ruminococcaceae.

Faecal microbiome taxonomic indicators of COPD using metagenomics. Having identified distinct COPD-associated faecal taxa using 16S rRNA gene sequencing, we sought to increase the resolution of these findings via metagenomic sequencing of the same samples. We recovered 437 metagenome-assembled 

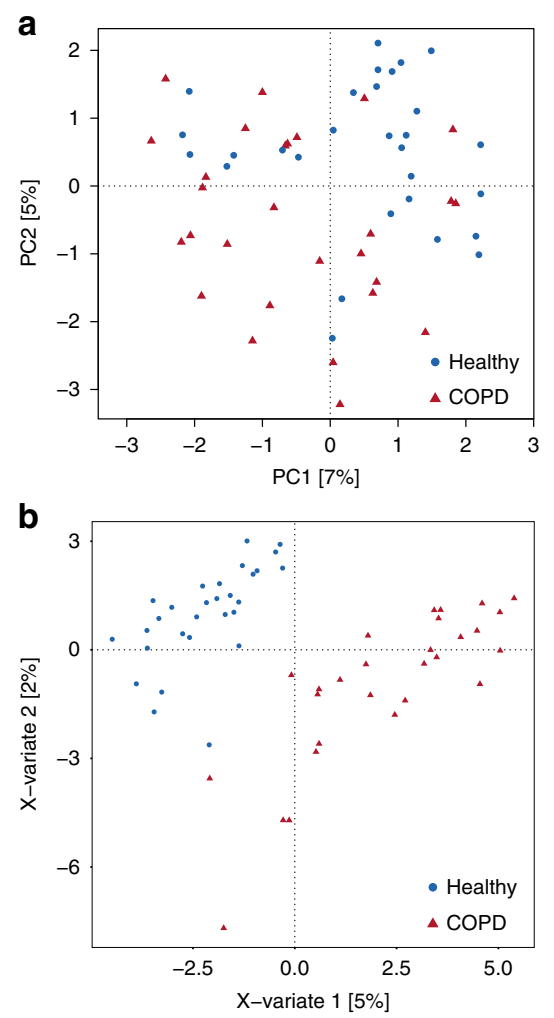

b

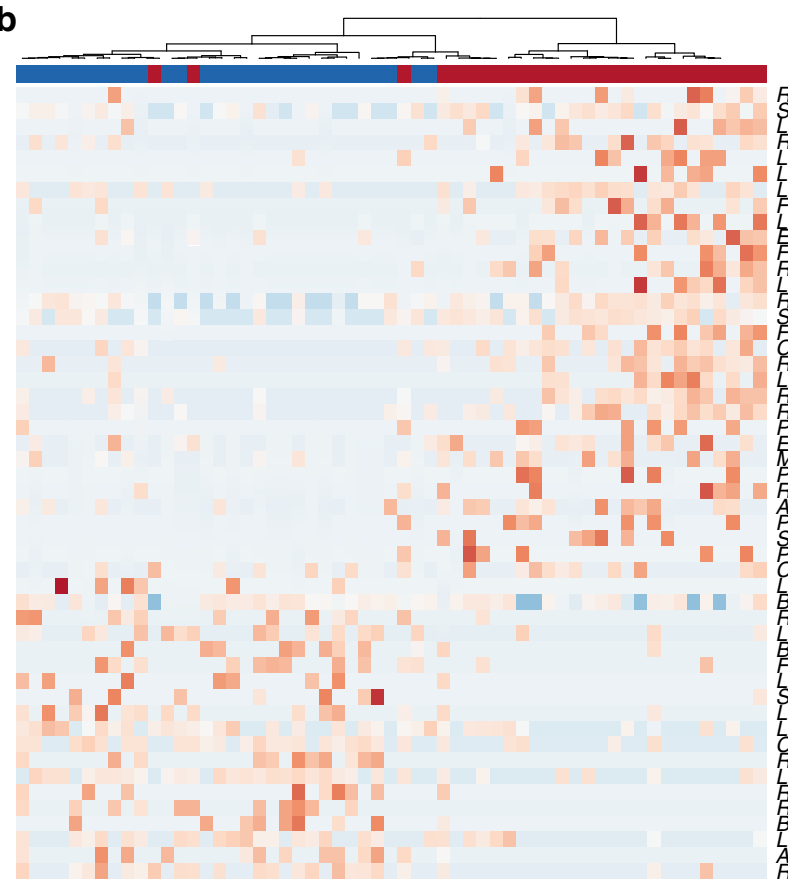

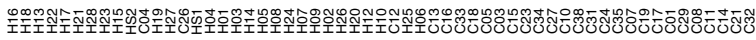

C

Clostridiaceae 1; Clostridium sensu stricto 1; b4a1da7442d103bac927d1ce0781e59 Lachnospiraceae; [Eubacterium] xylanophilum group; 1 ba1ebbb7da9918335ef52c875aec53f

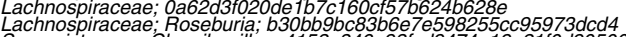
Synergistaceae: Cloacibacillus; $4153 a 84693$ fcd9474e19a21fod90500 Actinomycetaceae; Actinomyces; b59f842a087617c7cec2c79690dfedob Lachnospiraceae; 1e8b9381eaf7927036781243970760ł3 Ruminococcaceae; UCG-010; 799b509b8c5d0ec45b8abae460486b06 Bacteroidetes; Bacteroidia; Bacteroidales; Bacteroidaceae; Bacteroides; $11139544 a 2 b 85 e a c d b 525 c e a 7 c 2 c a f 3 a$ Lachnospiraceae; Lachnospira; 3 e427b8b661 b5991ecdd3088bb284e55 113054 Ruminococcaceae; Ruminiclostridium 5; 7033a395fdef3a1e16959bbde 12 fot Ruminococcaceae; NK4A214 group; 691b8e31532f61 fa7cd8dc6981ea7db Peptostreptococcaceae; Romboutsia; 8b86d0245fe5716eb303a46c9d10a4bo Ruminococcaceae; $62 b 76963$ e8f620ac6cad5cbc42f97c9f Ruminococcaceae; UCG-014; 8a50b1ddf55600c3b1585 Lachnospiraceae; 95e0459b3d40ł37ab68209c9256bc853 Ruminococcaceae; UCG-003; eaf7ae14055b033f41391df1895bf101 Peptostreptococcaceae; Intestinibacter; 35 t2a10479b3fb
Lachnospiraceae; d3f11 151fd34335a81dd3c77f12f1467 Lachnospiraceae, fbaff93b9e4e6d50d05b80b6ff89? Lachnospiraceae; Blautia; 540 b157ec4a927059d243309656634b9 Lachnospiraceae; Marvinbryantia; c071ecaafa203d59f6327d361e167bf4 Micrococcaceae; Rothia; aa46cb9fb30bcadedeed18629f585659
Ruminococcaceae; UBA1819; c34f0a17a3aa289ee978d0557bb6a0b4 Bacteroidaceae; Bacteroides; af02659f726560e4a8061 baagbafdoa Ruminococcaceae; UCG-005; 4ab6f76deade2f7e045f672e44dc8bd Family XIII; Family XIII AD 3011 group; 1 c974d1c24tbaaee6d4ea5fcade70e6 Streptococcaceae; Streptococcus; Streptococcus mutans; d41073c6e011b55c0a90d4f12ab22d3b

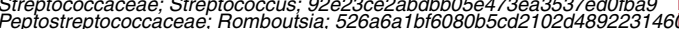
Lachnospiraceae; b6b6706232cd5f48a9d335bf738bc07d Christensenellaceae; R-7 group; Ocf6a037300dcdd808c0df570175587f Lggerthellaceae; 3 c6ccidolostridium; f8fa3cf231a92ada03642dec9efo71c1 Acidaminococcaceae; Phascolarctobacterium; 9c24ae9bb6ct6e5da1482706070a412c Family XIII AD3011 group; 6e58d361f19f169d10b112fbdeb48fc0 Rikenellaceae; Alistipes; 093521120b2a01c376e96b16ffd9a67
Ruminococcaceae; Ruminiclostridium 5; 5eff7ef7ad68f1t36e072816415ca439 Lachnospiae; [Eubacterium] eligens group; b409c6d40f705d51007c09065fc52ec6 Streptococcaceae; Streptococcus: bfe69754325dc5dacd606216a990d606 Ruminococcaceae; DTU089; fde69e55bbd05719012f99b275b556a2 Ruminococcaceae; e4b62ed7117e2ee682b3a3723ea0c2ce
Christensenellaceae; $R-7$ group; fa73e3189bcaef365aa0e08831a28e25

\begin{tabular}{|c|c|c|c|c|}
\hline-0.2 & -0.1 & 0.0 & 0.1 & 0.2 \\
\hline
\end{tabular}

Fig. 1 Faecal microbiota of COPD patients $(n=28)$ can be distinguished from that of healthy individuals $(n=29)$ using 165 rRNA gene amplicon sequencing. a Principal component (PC) analysis undertaken at the sequence variant level using read counts transformed using log-cumulative-sum scaling. b Multivariate sparse partial least-squares discriminant analysis (sPLS-DA) of read counts transformed using log-cumulative-sum scaling at the sequence variant level. c Sequence variants contributing to separation along with component 1 of sPLS-DA from $\mathbf{b}$. Bar length indicates loading coefficient weight of selected genomes, ranked by importance, bottom to top; bar colour indicates the group in which the sequence variant has the highest median abundance, red $=\mathrm{COPD}$, blue $=$ healthy. $\mathbf{d}$ Heatmap of read counts transformed using log-cumulative-sum scaling of discriminatory sequence variants identified along with component 1 of sPLS-DA from (b). 
genomes (MAGs) from 57 individuals, each with an estimated completeness $>80 \%$ and a maximum of $7 \%$ contamination. Overall community composition was analysed using these MAGs in combination with a set of publicly available reference genomes. Consistent with the 16S rRNA gene sequencing analysis, COPD and healthy samples could be distinguished (Supplementary Fig. 1a, $p<0.0001$, PERMANOVA of Bray-Curtis distances) despite considerable variation in community composition between individuals (Supplementary Fig. 1b) and no significant differences in diversity between the groups $\left(p_{\text {Shannon }}=0.174\right.$, $\left.p_{\text {SimpsonInverse }}=0.345\right)$. COPD status explained $6 \%$ of the between-sample variability (Supplementary Data 8). At the bacterial family level, Bifidobacteriaceae, Eubacteriaceae, Lactobacillaceae, Micrococcaceae, Streptococcaceae and Veillonellaceae were enriched in COPD. Depleted families included Desulfovibrionaceae, Gastranaerophilaceae and Selenomonadaceae along with several uncharacterised families of Bacilli and Clostridia (Supplementary Data 9). Enriched and depleted families were highly variable between individuals (Supplementary Data 9 and Supplementary Fig. 1b), as is frequently observed with human datasets ${ }^{29}$.

To identify genera and species contributing to the distinction between COPD and healthy controls, we employed both univariate and multivariate approaches designed to identify significantly different species (DESeq $2^{30}$ ) and the largest source of variation between the two groups (mixOmics ${ }^{31}$ ), respectively (Fig. 2a-c). Over 200 genomes belonging to 107 genera and 146 species were identified as either significantly enriched or depleted between COPD and healthy samples using DESeq2 although the differences in average relative abundance for most species were small (Supplementary Data 10). Some species were present at a substantially higher prevalence in COPD patients including Rothia and Streptococcus spp., Romboutsia timonensis and Intestinibacter bartlettii, consistent with 16S rRNA gene sequencing, while others were more prevalent in healthy controls (e.g. Coprobacter fastidiosus and Coprobacter secundus, Rikenellaceae genus RC9 and Christensenellales family CAG-74). Streptococcus species were identified as key differentiators between COPD and healthy samples using sPLS-DA analysis within mixOmics, as were multiple members of the family Lachnospiraceae (Fig. 2b, c).

Microbiome changes indicate disease status. To test whether patient characteristics contributed to the microbiome signature separating COPD from healthy controls, we repeated the univariate analysis of the metagenomic data, including age, BMI and sex within a multifactorial design in DESeq2, categorising BMI according to WHO standards and age in 10 -year windows $(\leq 54$, $55-64,65-74$ and 275$)$. Streptococcus vestibularis, and two unnamed Streptococcus species (sp001556435, sp000187445) remained significantly enriched in COPD samples using this model, and RC9 genomes remained enriched in healthy samples (Supplementary Data 11). We compared medication-related subgroups within the COPD samples and found no significant difference in microbiome composition between those taking inhaled steroids, beta-agonists or anticholinergics and those not taking these drugs $(p=0.286,0.208$ and 0.220 , respectively, PERMANOVA of Bray-Curtis distances). There was also no significant difference between current smoking and non-smoking COPD patients $(p=0.224$, PERMANOVA of Bray-Curtis distances) or between stable and frequent exacerbators $(p=0.367$, PERMANOVA of Bray-Curtis distances). Correlation analysis revealed a subset of taxa that were significantly associated with lung function. These included negative correlations between Streptococcus sp000187445 and $S$. vestibularis and forced expiratory volume in $1 \mathrm{~s}\left(\mathrm{FEV}_{1}\right)$ and most COPD-associated members of the family Lachnospiraceae with predicted per centforced vital capacity (FVC) and $\mathrm{FEV}_{1}$ (Fig. 3). Positive correlations were observed between Desulfovibrio piger_A and CAG-302 sp001916775 and lung function. Overall, these data support an association between the faecal microbiome and COPD status, identifying species associated with both health and disease; there are some associations with disease severity, as indicated by blood neutrophils, lung function and historical frequency of exacerbation episodes.

Functional potential indicators of the COPD faecal microbiome. Metagenomic reads were annotated with predicted function based on alignment against available databases (Pfam, TIGRFAM, KEGG and CAZy), for a gene-centric analysis of unassembled metagenomes. There was no significant difference in overall predicted functional capacity between COPD and healthy samples in a global comparison of all annotated domains (Supplementary Fig. 2). However, pairwise comparison at the individual domain level revealed several annotated functions that were distinct between the two groups. Glucosyltransferase enzymes were enriched in COPD based on enrichment of domains in each database: PF02324 (Pfam), TIGR04035 (TIGRFAM), K00689 (KEGG) and GH70 (CAZy) (Supplementary Data 12-15). These enzymes synthesise high-molecular-weight extracellular glucan polymers such as $\alpha$-D-glucans from sucrose that adsorb onto the bacterial surface and contribute to the adherence of Streptococcus and other species $^{32}$. LPXTG-anchored adhesion domains (K12472 and TIGR04225), a cell-surface-anchoring motif found in Gram-positive bacteria, were also enriched in COPD samples. Most of the reads annotated as containing the enriched domains aligned to the enriched Streptococcus populations (Supplementary Data 16-19). Glucosyltransferase-annotated reads aligned to $S$. salivarius and Streptococcus sp001556435 gtfC genes, of which there are multiple copies within the enriched reference genomes. LPXTG-anchored adhesion domains were identified within a YSIRK-type signal peptide-containing protein in S. salivarius, $S$. parasanguinis_B and other Streptococcus spp. (Supplementary Data 20 and 21). The protein also carries multiple CshA-type fibril repeats used by Streptococcus gordonii to bind fibronectin ${ }^{33}$. Fibronectin is expressed by epithelial cells and is upregulated in murine models of colitis and in association with inflammatory bowel disease $\mathrm{s}^{34,35}$. Increased fibronectin is observed in the small airways of COPD patients ${ }^{36}$ and in experimental $\mathrm{COPD}^{37}$; however, no similar analysis is available for the gut. The capacity for adhesion to host tissue may therefore contribute to the enrichment of streptococci in the COPD gut microbiome.

We then also undertook a targeted genome-centric analysis comparing the encoded functions within genomes identified as significantly different between COPD and healthy samples in either multivariate or covariate-adjusted univariate analyses (35 enriched in, and 25 depleted in COPD relative to healthy controls, Fig. $2 \mathrm{~b}$ and Supplementary Data 11). The majority of the predicted discriminatory functions were encoded in genomes enriched in COPD (Supplementary Data 22). These included Streptococcus-specific features such as the accessory secretory proteins Asp1-3, forming part of the accessory SecA2/ Y2 secretion system that exports glycosylated serine-rich repeat glycoproteins involved in adhesion ${ }^{38}$. Also specific to Streptococcus are the typical streptococcal peptidoglycan biosynthesis enzymes (penicillin-binding proteins, murN) and an ABC-type manganese uptake system involved in streptococcal virulence ${ }^{39}$. Elements of multiple amino acid biosynthesis pathways were also enriched among COPD-associated genomes, as were fatty acid biosynthesis initiation and elongation enzymes. Genomes 


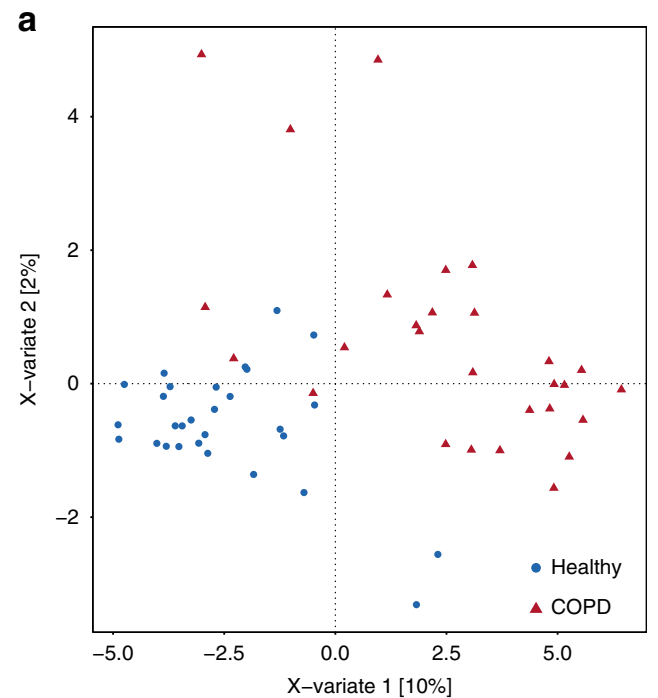

b GCF 002159845.1: Acutalibacteraceae; Anaeromassilibacillus sp002159845

GCA 002073515.1; Streptococcaceae; Streptococcus sp001556435 SB1789 bin. 16; Ruminococcaceae; CAG-115 sp003531585 GCF_000164675.2; FStreptococcaceae; Streptococcus parasanguinis GCF_002160305.1; Oscillospiraceae; Lawsonibacter sp002160305 GCF_002160385.1; Butyricicoccaceae; Butyricicoccus pullicaecorum GCF 000010145.1; Lactobacillaceae; Lactobacillus $H$ fermentum GCF 002160985.1: Lachnospiraceae; Dorea sp002160985 GCF 000314795 2. Streptococcaceae. Streptococcus spo00314795

GAF 000314795.2, Strep SA9249_bin. 11; Ruminococcaceae, Rulhenibacterium GCF_001809565.1; Micrococcaceae; Rothia mucilaginosa_A GCF_000180075.1; Streptococcaceae; Streptococcus vestibularis SA9256_bin. 12; Lachnospiraceae; Faecalicatena sp002314255 GCF_000262145.1; Streptococcaceae; Streptococcus parasanguinis GCF 002160015.1: Ruminococcaceae: Anaerofilum sp002160015 GCF 001813525.1; Streptococcaceae; Streptococcus parasanguinis B GCA 900067065.1; Acutalibacteraceae; Marseille-P4683 sp900232885 GCF_900142165.1; Lachnospiraceae; Hespellia stercorisuis GCF_000509025.1; Lachnospiraceae; Sellimonas intestinalis GCF_002160825.1; Lachnospiraceae; GCA-900066575 sp002160825 GCF_001812775.1; Micrococcaceae; Rothia mucilaginosa_A GCF_000187445.1; Streptococcaceae; Streptococcus sp000187445 GCF 001546835.1: Streptococcaceae; Streptococcus sp000187445 GCF 001754075.1: Lachnospiraceae; Dorea faecis

GCF 002159935.1: Eggerthellaceae. Rubneribacter

GCF_002159935.1; Eggerthellaceae; Rubneribacter badeniensis GCF_002161065.1; Lachnospiraceae; Eubacterium_E sp002161065 GCF_002159835.1; Lachnospiraceae; Blautia_A sp002159835 GCF_900120155.1; Lachnospiraceae; Faecalicatena sp90012015 GCF 002161525.1: Lachnospiraceae; Sellimonas sp002161525 GCF 900155415.1 ; Christensenellaceae; Christensenella massiliensis

0.0

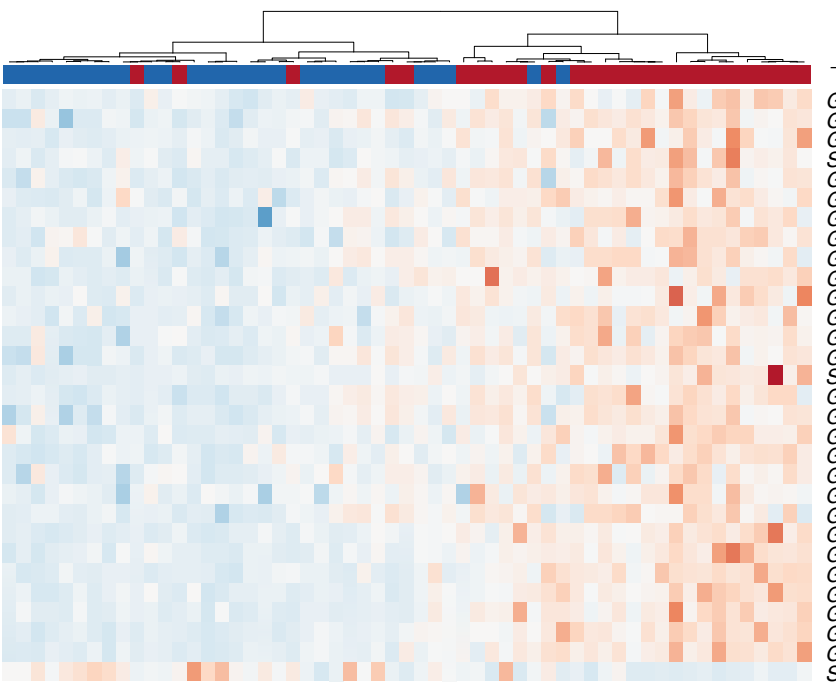
$-4.73-2.36 \quad 0 \quad 2.36 \quad 4.73 \quad$ COPD

GCF_002159845.1; Acutalibacteraceae; Anaeromassilibacillus sp002159845 GCF 000164675.2; Streptococcaceae; treptococcus parasanguinis GCF 000509025.1; Lachnospiraceae; Sellimonas intestinalis SA9249 bin.11; Ruminococcaceae; Ruthenibacterium GCF 000314795.2; Streptococcaceae; Streptococcus sp000314795 GCF_002160385.1; Butyricicoccaceae; Butyricicoccus pullicaecorum GCF_001546835.1; Streptococcaceae; Streptococcus sp000187445 GCA 900067065 .1; Acutalibacteraceae; Marseille-P4683 sp900232885 GCF 000180075.1; Streptococcaceae; Streptococcus vestibularis GCF 002159935.1; Eggerthellaceae; Rubneribacter badeniensis GCF 002160305.1 ; Oscillospiraceae; Lawsonibacter sp002160305 GCF-900142165.1; Lachnospiraceae; Hespellia stercorisuis GCF_001812775.1; Micrococcaceae; Rothia mucilaginosa_A GCF 000262145.1 ; Streptococcaceae; Streptococcus parasanguis SA9256 bin.12; Lachnospiraceae; Faecalicatena sp002314255 GCF 001813525.1; Streptococcaceae; Streptococcus parasanguinis GCF 002160015.1; Ruminococcaceae; Anaerofilum sp002160015 GCF 900155415.1; Christensenellaceae; Christensenella massiliensis GCF_001809565.1; Micrococcaceae; Rothia mucilaginosa_A GCF_000010145.1; Lactobacillaceae; Lactobacillus_H fermentum GCA O02073515.1; Streptococcaceae; Streptococcus sp001556435 GCF 002161525. 1; Lachnospiraceae; Sellimonas sp002161525 GCF_002160825.1; Lachnospiraceae; GCA-900066575 sp002160825 GCF-900120155.1; Lachnospiraceae; Faecalicatena sp90012C GCF 002160985.1; Lachnospiraceae; Dorea sp00216

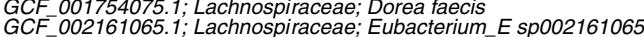
GCF 002159835.1; Lachnospiraceae; Blautia A spo0 02159835

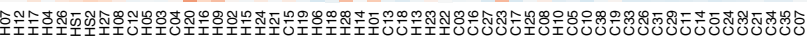
SB1789_bin.16; Ruminococcaceae; CAG-115 sp003531585

Fig. 2 Metagenomic sequencing-based exploration of COPD-associated $(n=28)$ faecal microbiomes supports distinction from those of healthy individuals $(\boldsymbol{n}=\mathbf{2 9})$. a Multivariate sparse partial least-squares discriminant analysis (sPLS-DA) of read-mapping-based relative abundance at the genome

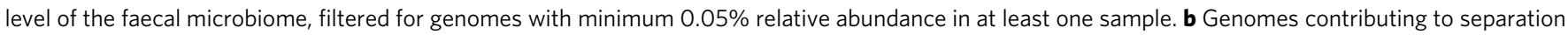
along with component 1 of sPLS-DA from (a). Bar length indicates loading coefficient weight of selected genomes, ranked by importance, bottom to top; bar colour indicates the group in which the genome has the highest median abundance, red = COPD, blue = healthy. $\mathbf{c}$ Heatmap of discriminatory genomes along component 1 of sPLS-DA from (a). Data are centred with log-ratio-transformed relative abundance.

associated with healthy samples from the uncharacterised families CAG-138 (order Christensenellales), CAG-239 (order RF32), CAG1000 (order RF39), CAG-302 (order RF39) and CAG-508 (order TANB77) lack many of these functions based on KEGG module completeness (Supplementary Data 23), as recently observed amongst uncultivated members of the gut microbiome ${ }^{40}$. They may therefore represent gut symbionts reliant on host metabolites making them potentially more sensitive to environmental perturbation.

Functional indicators of the COPD faecal metabolome. To assess metabolic expression in the COPD gut, we undertook untargeted metabolomic profiling of paired faecal samples identifying 934 compounds likely arising from both the microbiome and the host, and some from ingested compounds
(Supplementary Data 24). Principal component analysis (PCA, Supplementary Fig. 3a) revealed significant but incomplete separation of COPD and healthy samples $(p=0.003$, PERMANOVA of Euclidean distances). As with the metagenome, there was no significant difference in the metabolome of COPD patients between those taking steroids, beta-agonists or anticholinergics and those not $(p=0.299,0.724$ and 0.596 , respectively, PERMANOVA of Euclidean distances), between current smokers and non-smokers $(p=0.115)$, or stable and frequent exacerbators $(p=0.501)$.

Integration of metagenomes and metabolomes. We used the mixOmics platform to both investigate the metabolites contributing to the distinction between COPD and healthy samples and to integrate the metagenomic and metabolomic data into a 


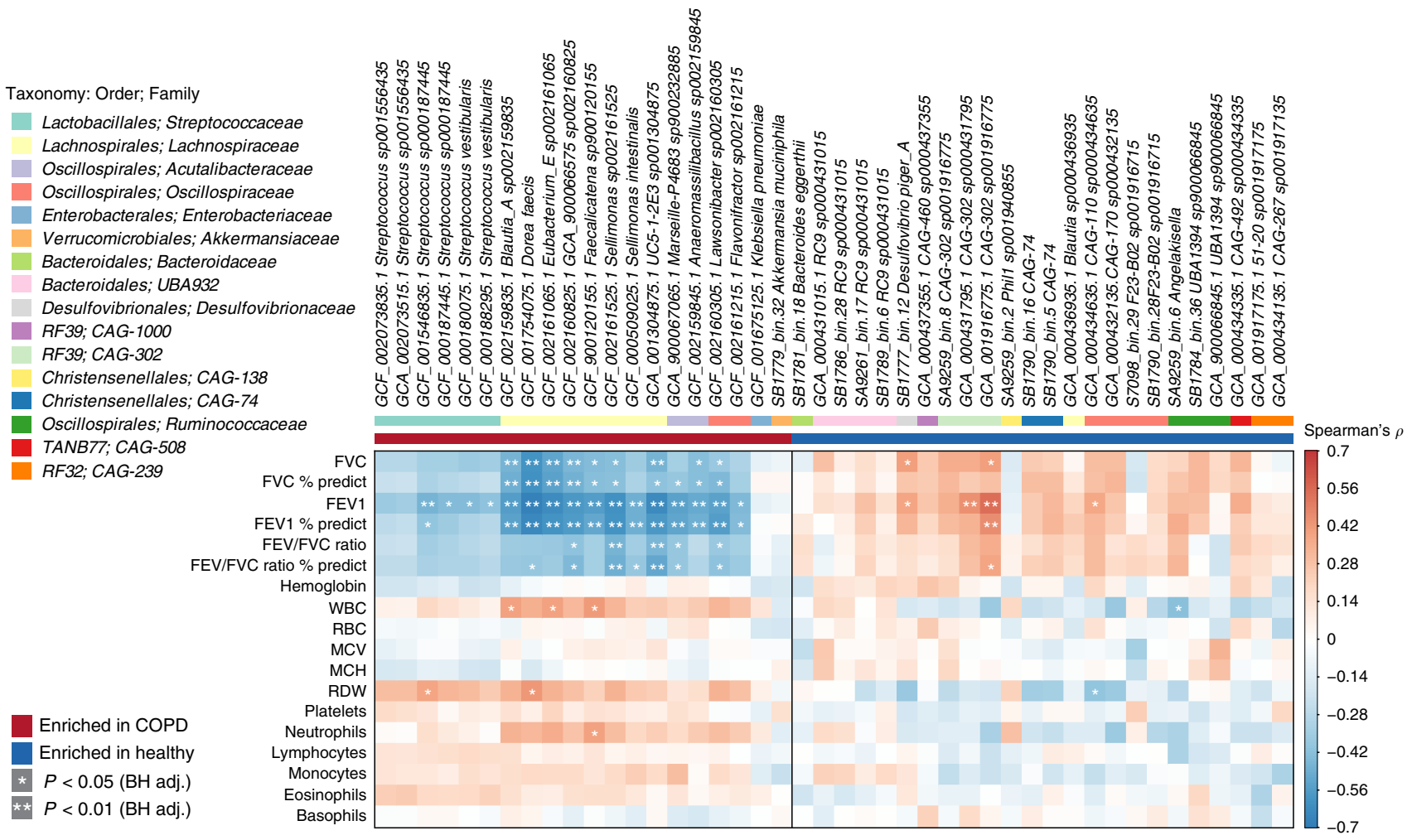

Fig. 3 Correlation of members of the faecal microbiome with lung function. Spearman's rho calculated between mapping-based read counts per genome and phenotypic scores. Genomes included are those from Supplementary Data 11, with enrichment in either COPD or healthy samples indicated by the coloured bar along the top of the heatmap. White stars within heatmap boxes indicate significant results $\left({ }^{\star} p<0.05\right.$; ${ }^{\star \star} p<0.01$, Student's $t$ test (two-sided), Benjamini-Hochberg adjustment for multiple comparisons. Exact $p$ values are provided in Supplementary Data 34). Genome abundances were centred with log-ratio transformation prior to analysis. FVC forced vital capacity, FEV forced expiratory volume, WBC white blood cell, RBC red blood cell, MCV mean corpuscular volume, MCH mean corpuscular haemoglobin, RDW red cell distribution width. COPD: $n=28$; healthy: $n=29$.

multi-omic signature (Fig. 4 and Supplementary Fig. 3b). Analysis of species confirmed enrichment of $S$. parasanguinis_B and $S$. salivarius in association with COPD (Fig. 4a, b). Within the metabolome, COPD samples were largely defined by depletion of metabolites, with $76 \%$ of the identified signature being metabolites present at higher abundance in healthy samples (Fig. $4 \mathrm{c}, \mathrm{d}$ and Supplementary Data 25). Of the top 50 indicator metabolites separating COPD from healthy samples, $46 \%$ were from the lipid $(n=23), 20 \%$ amino acid $(n=10)$ and $20 \%$ xenobiotic $(n=10)$ classes (Supplementary Data 25), indicating that lipid metabolism may be altered in COPD. Sixteen of these compounds, all from the lipid, amino acid or xenobiotic classes, were identified as significantly differential between COPD and healthy samples following adjustment for covariates (age, sex and BMI) using a linear model (Supplementary Data 25). Correlation analysis between the 44 bacterial genomes identified above (Supplementary Data 11) and these 16 metabolites revealed 253 significant associations, many of which involved species enriched in COPD (Fig. 5).

Lipid involvement in the COPD faecal metabolome. Within the lipid class, all six metabolites identified as significant in the linear model were enriched in healthy samples (Supplementary Data 25). Four of these were the dicarboxylic acids suberate (C8), sebacate $(\mathrm{C} 10)$, undecanedioate $(\mathrm{C} 11)$ and dodecanedioate $(\mathrm{C} 12)$ that may originate from the diet or be produced endogenously via the $\omega$-oxidation of fatty acids ${ }^{41,42}$. Each of these four lipid metabolites was negatively associated with the majority of species enriched with COPD, suggesting possible 'guilt-by-association' related to the COPD versus healthy divide (Fig. 5). In contrast, only a subset of species enriched in healthy samples was positively associated with the four dicarboxylates. Bacterial catabolism of dicarboxylic acids has been described in vitro ${ }^{43}$; therefore, we looked for the described enzymes within the genomes of the enriched and depleted species (Supplementary Data 11). While some species are potentially capable of degrading dicarboxylic acids, the pattern of enzyme presence did not match the observed associations with species abundance, either within the healthy or COPD samples (Fig. 5, Supplementary Data 26), supporting a human-derived component of the phenotype. Since the use of statins can influence the rate of fatty acid oxidation ${ }^{44}$, we added statin use to the linear model described above. Dicarboxylic acids were no longer significantly depleted in COPD samples following this adjustment (Supplementary Data 25), indicating that statin medication may be driving this phenotype. Inclusion of additional medication (proton-pump inhibitors, selective serotoninreuptake inhibitors, beta-blockers, angiotensin-converting enzyme inhibitors and angiotensin II receptor antagonists) in an extended linear model reduced the number of significant metabolites to five: amino acid metabolites $\mathrm{N}$-acetylglutamate and $\mathrm{N}$-acetylproline and the xenobiotic metabolites cotinine, asmol and $\mathrm{N}$-carbamoylglutamate, again implicating medication use as impacting the levels of other metabolites.

Amino acid involvement in the COPD faecal metabolome. Without adjustment for medication, two amino acid metabolites were enriched and three were depleted in COPD. The first enriched metabolite, $\mathrm{N}$-acetylcadaverine, has previously been associated with Crohn's disease ${ }^{45}$. The precursor of $\mathrm{N}$-acetylcadaverine, cadaverine, is formed during lysine degradation; however, cadaverine levels 

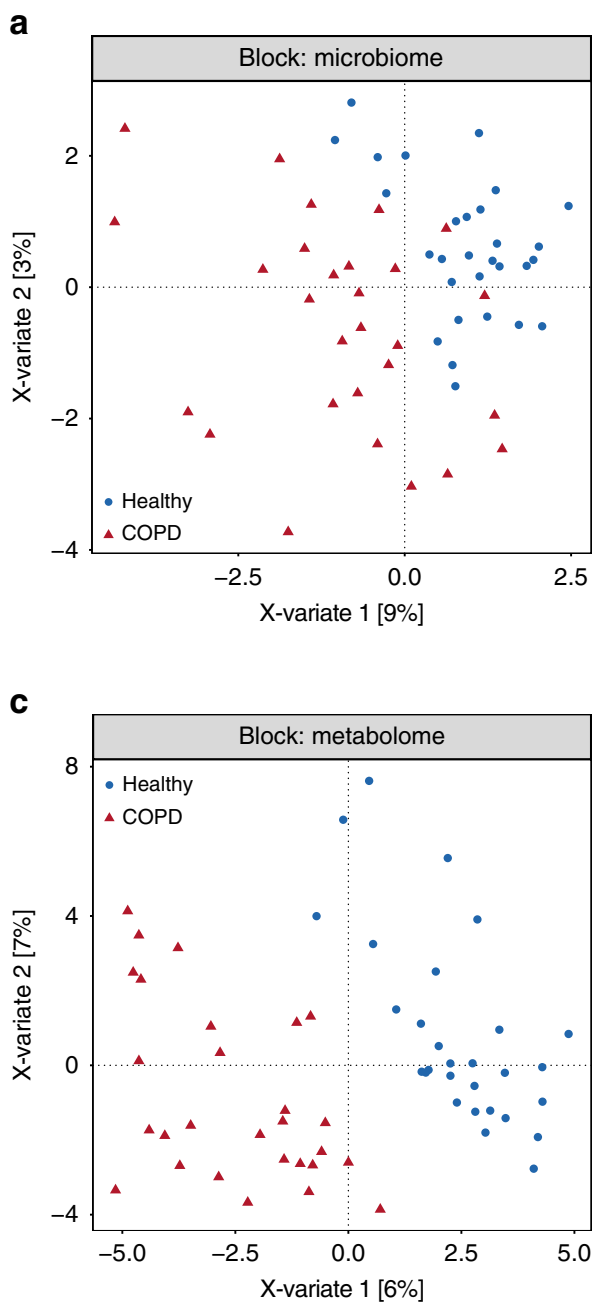

b

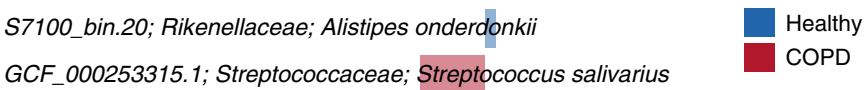

GCF_002159845.1; Acutalibacteraceae; Anaeromassilibacillus sp002159845

SA9249_bin.11; Ruminococcaceae; Ruthenibacterium

GCF_001813525.1; Streptococcaceae; Streptococcus parasanguinis_B

\begin{tabular}{cccccc}
\hline & 1 & 1 & & & \\
-0.8 & -0.6 & -0.4 & -0.2 & 0 & 0.2 \\
& \multicolumn{6}{c}{ Loading }
\end{tabular}

d

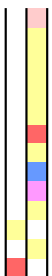

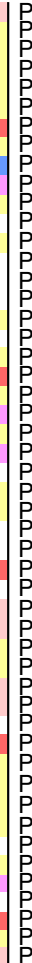

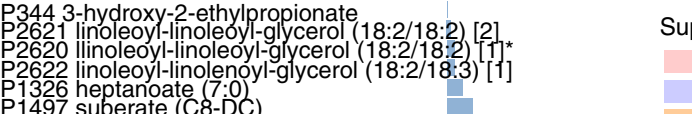

P 1497 suberate $(C 8-D C$ -
P

P1376 heptadecatrienoate $(17: 3)^{*}$

P3932 pantoate

P 3756 adenosine $($ S10-DC)

(1774 3-hydroxysebacate

P1778 3-hydroxystearate

P4152 cotinine

P1195 matyryl-leucine

P2018 mannose

P5283 N-propionylmethionine

P1358 oleate/vaccenate $(18: 1)$

P4450 nicotianamine

P1507 dodecanedioate (C12)

P1504 undecanedioate (C11-DC)

P407 N-acetyltaurine

P1629 acetyltarnitine (C2)
P2548 1-linolenoylglycerol (18:3)

P2548 1-linolenoylglycerol (18:3)

P4704 2-acetamidobutanoate

P4545 ursolic acid

P123 6-oxopiperidine-2-carboxylate

P449 argininate ${ }^{\star}$ P2414 1-stearoyl-GPI (18:0)

P2414 1-stearoyl-GPI (18:0)
P1391 linoleate (18:2n6)

P434 N-acetylproline

P434 N-acetylproline

P125 N-acetyl-cada

P4566 harmane $(18: 3 n 3$ or $3 n 6)$

P2411 1-palmitoyl-GPI* (16:0)

P1976 glycerophosphoinositol

P2404 1-palmitoyl-GPG (16:0)

P2541 1-pentadecanoylglycerol (15:0)

P3778 2'-deoxyadenosine
55104 asmol
55127 N-carbamoylglutamate

P5127 N-carbamoylglutamate
P2675 2-palmitoyl-galactosylglycerol (16:0)*
P45 N-acetylglutamate

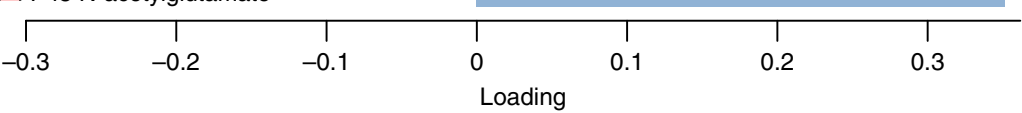

Fig. 4 Faecal metabolome of COPD patients $(n=28)$ is distinguished from that of healthy individuals $(n=29)$ using a multi-omic analysis. a $D I A B L O$ sample plot demonstrating discrimination between COPD and healthy samples based on microbiome data. $\mathbf{b}$ Genomes contributing to separation along with component 1 of (a). Bar length indicates loading coefficient weight of selected genomes, ranked by importance, bottom to top; bar colour indicates the group in which the sequence variant has the highest median abundance, red =COPD, blue = healthy. Microbiome data are centred log-ratio-transformed relative abundance, filtered for genomes with minimum $0.05 \%$ relative abundance in at least ten samples. c DIABLO sample plot demonstrating discrimination between groups based on metabolomics data. $\mathbf{d}$ Metabolites contributing to separation along with component 1 of (c). Metabolome data are log-transformed median-scaled values with missing values imputed using the minimum value for each compound, filtered for metabolites returning measurements in at least ten samples.

were not significantly different between COPD and healthy samples (Supplementary Data 25). Microbial production of $\mathrm{N}$ acetylcadaverine has been reported in the soil bacterium Corynebacterium glutamicum $^{46}$; however, we did not observe any positive associations between the metabolite and COPD-associated species (Fig. 5), and only one species, Rothia mucilaginosa_A, is predicted to carry the $\mathrm{N}$-acetyltransferase required for its production (Supplementary Data 26). The second enriched amino acid metabolite, $\mathrm{N}$-acetyltaurine, can be produced endogenously from taurine; however, there was no significant difference in taurine levels between COPD and healthy samples (Supplementary Data 25). In urine, elevated levels of $\mathrm{N}$-acetyltaurine are used as a marker of ethanol metabolism ${ }^{47}$; however, it is unclear what the biological significance is in faeces. Alcohol consumption was also significantly lower in COPD patients (Supplementary Data 27). The capacity to use $\mathrm{N}$-acetyltaurine as a carbon source has been described in several marine bacteria ${ }^{48}$ and, while we identified homologues of an $\mathrm{N}$-acetyltaurine $\mathrm{ABC}$ transporter in the majority of genomes associated with both COPD and healthy samples, only two, Anaeromassilibacillus sp002159845 and Lachnospiraceae GCA900066575 sp002160825, encoded homologues of the amidohydrolase required for converting $\mathrm{N}$-acetyltaurine to taurine (Supplementary Data 26). Both amidohydrolase-encoding species positively correlated with the abundance of $\mathrm{N}$-acetyltaurine, although they were not the only species displaying this trend (Fig. 5).

Of the three depleted amino acid metabolites in COPD without adjusting for medication, $\mathrm{N}$-acetylglutamate, $\mathrm{N}$-acetylproline and 6-oxopiperidine-2-carboxylate, the first two were also significantly depleted in the extended linear model (Supplementary Data 25). N-acetylglutamate is both a human and microbialderived metabolite, and may also be ingested ${ }^{49}$. In humans, 


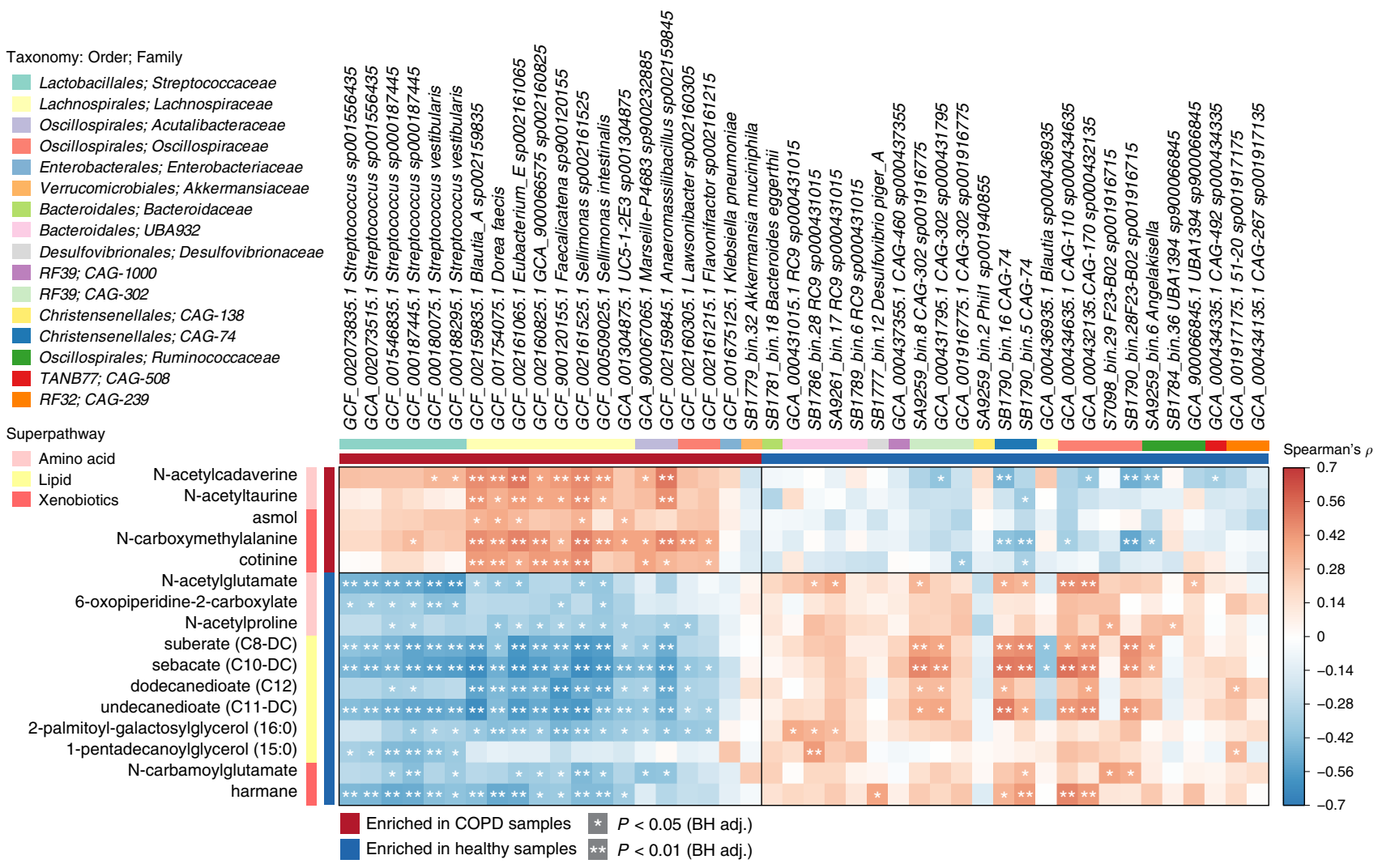

Fig. 5 COPD-associated species correlate with metabolites differentiating COPD $(\boldsymbol{n}=\mathbf{2 8})$ and healthy $(\boldsymbol{n}=\mathbf{2 9})$ individuals. Species and metabolites included are those identified as significantly differential between COPD and healthy samples, including age, sex and BMI within the relevant models (Supplementary Data 7 and 21). Enrichment in either group indicated by coloured bars to the left and top of the plot. Significant correlations denoted by white stars $\left({ }^{\star} p<0.05 ;{ }^{\star \star} p<0.01\right.$, Student's $t$ test (two-sided), Benjamini-Hochberg adjustment for multiple comparisons. Exact $p$ values are provided in Supplementary Data 35). Higher taxonomy of species (order, family) and super pathway of metabolites are indicated by coloured bars.

$\mathrm{N}$-acetylglutamate functions as a cofactor for carbamoyl phosphate synthetase I, the first enzyme in the urea cycle, while in bacteria, it is the first intermediate in the arginine biosynthetic pathway ${ }^{50}$. No other elements of the urea cycle were identified as significant (Supplementary Data 25). The majority of genomes enriched in COPD encode $\mathrm{N}$-acetylglutamate synthase, necessary for the generation of $\mathrm{N}$-acetylglutamate from glutamate, versus five of the genomes enriched in healthy samples (Supplementary Data 26). This suggests that the increased abundance of the metabolite in healthy samples may be a product of endogenous metabolism or altered dietary intake. The role of the other two amino acid metabolites enriched in healthy samples is unclear. Nacetylproline has been associated with the consumption of processed protein ${ }^{51}$ and may therefore relate to diet. 6oxopiperidine-2-carboxylate is a by-product of penicillin production by Penicillium chrysogenum ${ }^{52}$.

Xenobiotic involvement in the COPD faecal metabolome. Within the xenobiotic class, metabolites increased in COPD include the tobacco metabolite cotinine and the respiratory drug salbutamol (asmol), the usage of which was reported by $70 \%$ $(n=20)$ of patients (Supplementary Data 2). Both cotinine and salbutamol remained significant in the extended linear model (Supplementary Data 25). Depleted xenobiotic metabolites, Ncarbamoylglutamate and harmane, both have potential beneficial effects in the gut. $\mathrm{N}$-carbamoylglutamate is an analogue of $\mathrm{N}$ acetylglutamate and has beneficial roles in the animal gut following supplementation, including stimulating arginine synthesis $^{53}$, protection against oxidative stress ${ }^{54}$ and epithelial cell proliferation ${ }^{55}$. However, its source within the human gut is unknown. The $\beta$-carboline alkaloid harmane is found in plants and is also a bacterial metabolite ${ }^{56}$ and may therefore have multiple origins in the gut. Harmane has antimicrobial properties $^{57}$ and may modulate the innate immune system ${ }^{58}$.

A disease-associated network in COPD. We also undertook network analysis based on the integration of metabolomics and metagenomic datasets using species and metabolites identified in $>10$ samples, as described above (Fig. 4), to look for associations between the broader microbiome and COPD-linked metabolites. Three distinct microbiome/metabolite clusters were defined (Fig. 6). The first indicated associations between S. parasanguinis_B, Ruthenibacterium sp. and Anaeromassilibacillus sp002159845 and a group of 13 metabolites (Fig. 6a), each identified as different between COPD and healthy samples in our multivariate analysis (Fig. 4d). Eight of these were also discriminatory following adjustment for age, sex and BMI; one enriched and seven depleted in COPD (Supplementary Data 25). The second and third networks do not contain any nodes enriched in COPD or healthy samples and therefore likely represent interactions additional to a disease state (Fig. 6b, c). The first cluster, therefore, represents a shortlist of disease-associated species and metabolites for future testing in clinical models.

Enrichment of Streptococcaceae family members in the COPDassociated gut microbiome is replicated in an independent validation cohort. To validate our microbiome findings, we undertook metagenomic sequencing of a validation cohort 


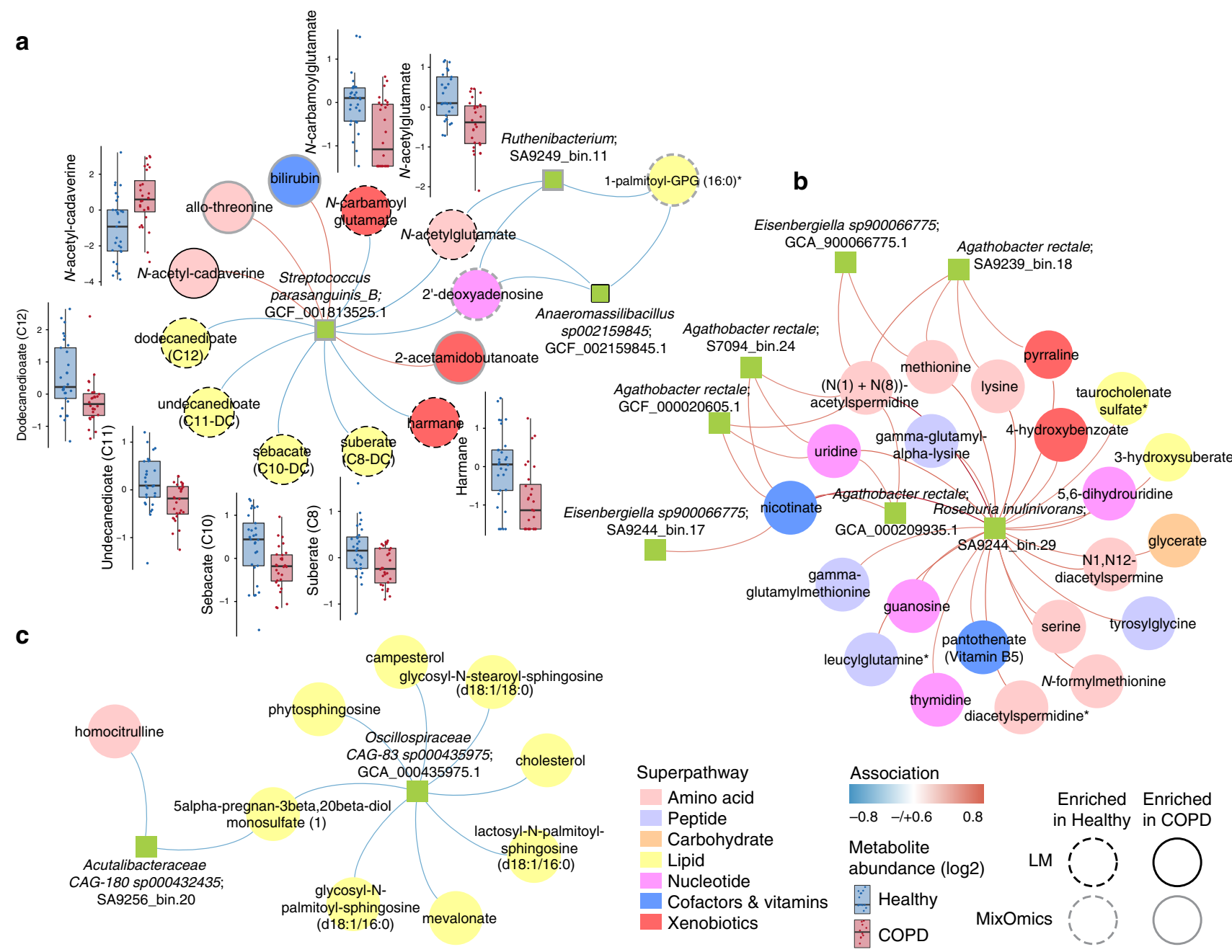

Fig. 6 Integration of faecal microbiomes and metabolomes identifies a COPD-associated network. a-c Integration of microbiome and metabolome datasets using the software DIABLO produced association networks showing correlations between bacterial species and metabolites. A positive correlation between nodes is indicated by red connecting lines, negative correlation by blue. Species and metabolites enriched in COPD or healthy samples are denoted by solid or dashed borders, respectively. Black borders indicate significance in the linear model adjusted for age, sex and BMI ( $p<0.05$, Wald test (two-sided) with Benjamini-Hochberg adjustment for multiple comparisons, Supplementary Data 7 and 21) and grey borders indicate selection by MixOmics as discriminatory along with component 1 of Fig. 4a, b. All metabolites significant within the linear model were also selected by MixOmics. The abundance of metabolites (log-transformed median scaled) significant in the linear model provided as boxplots adjacent to the relevant nodes. Each box centres on the median, with lower and upper bounds representing the first and third quartile (25th and 75th percentile), respectively. Whiskers extend 1.5 times the interquartile range from the outer bounds. Microbiome data filtered for genomes with minimum of $0.05 \%$ relative abundance in $\geq 10$ samples. Metabolome data filtered for metabolites returning measurements in $\geq 10$ samples. Microbiome data are centred log-ratio- transformed relative abundance. Metabolomics data are log-transformed median-scaled values with missing values imputed using a minimum value for each compound. COPD: $n=28$; healthy: $n=29$.

comprising 38 samples, 16 COPD patients and 22 healthy individuals (Supplementary Data 31). As with the study cohort, COPD and healthy stool samples could be distinguished based on bacterial community profiles $(p=0.037$, PERMANOVA of Bray-Curtis distances), with COPD status explaining $\sim 4 \%$ of between-sample variability (compared to $6 \%$ in the study cohort). Of the 210 genomes identified as enriched in either COPD or healthy samples in the study cohort (Supplementary Data 10), 59 (28\%) displayed a similar enrichment trend in the validation cohort of which $33(16 \%)$ reached significance including six of the Streptococcus spp. enriched in COPD samples, and RC9 spp., CAG-302 spp. and UBA11524 sp000437595 enriched in healthy samples (Supplementary Data 32 ). Using a multivariate approach,
$11(37 \%)$ of the 30 genomes identified as key differentiators of COPD and healthy samples in the study cohort were in the top 30 separating the groups in the validation cohort (Fig. 7a, b). Along with Streptococcus parasanguinis_B, highlighted in the disease-associated network (Fig. 6), these species included Eubacterium_E sp002161065, Sellimonas spp., Anaeromassilibacillus sp002159845 and Lawsonibacter sp002160305 that correlated with lung function in the study cohort (Fig. 3). At the functional level, six of the eight domains significantly enriched in COPD samples (Supplementary Data 8-11) followed a similar trend in the validation cohort, although none significantly so (Supplementary Data 33). This indicates that larger cohorts may be required to clearly differentiate COPD samples based on gut 

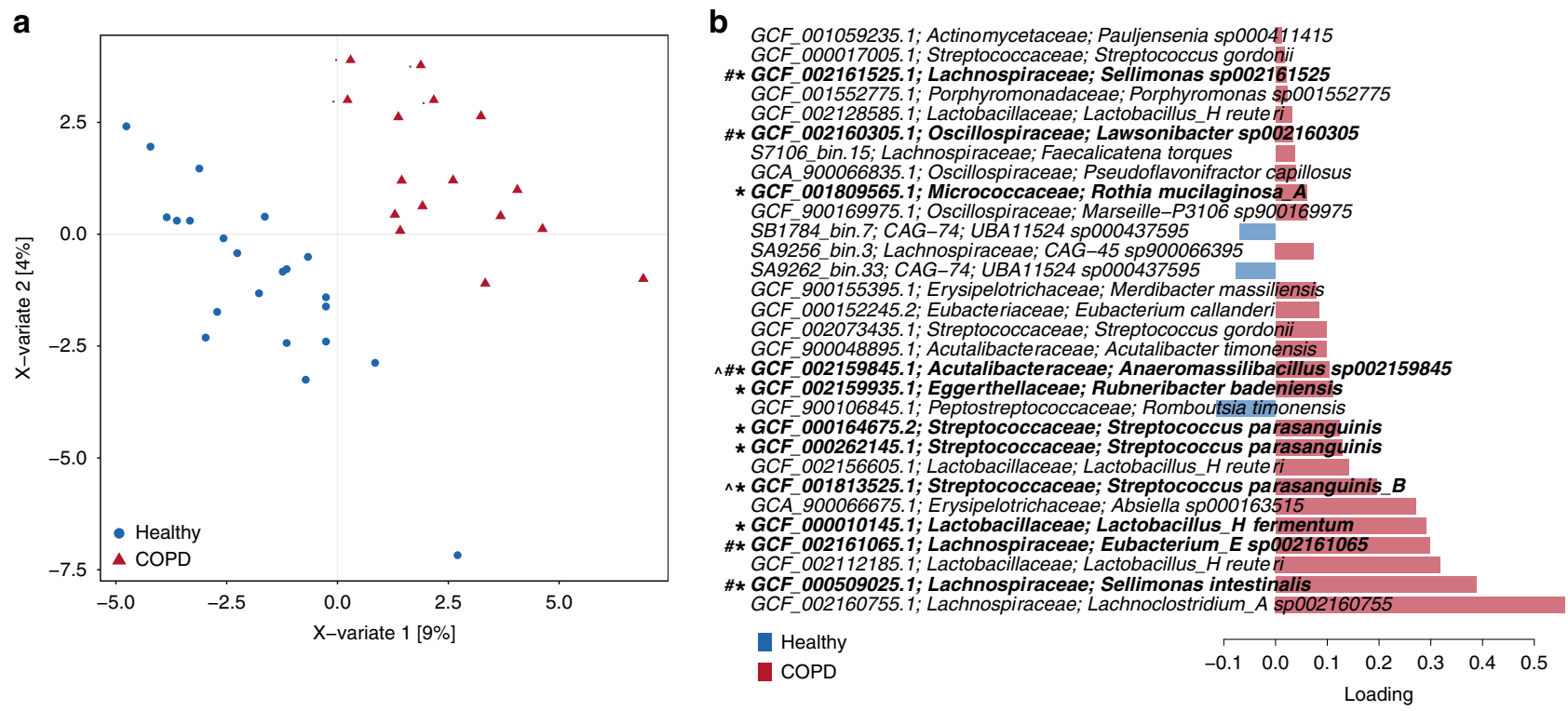

Fig. 7 Association of gut microbiome members with COPD replicate in an independent cohort. a Multivariate sparse partial least-squares discriminant analysis (sPLS-DA) of read-mapping-based relative abundance at the genome level of the faecal microbiome, filtered for genomes with minimum $0.05 \%$ relative abundance in at least one sample. $\mathbf{b}$ Genomes contributing to separation along with component 1 of sPLS-DA from (a). Bar length indicates loading coefficient weight of selected genomes, ranked by importance, bottom to top; bar colour indicates the group in which the genome has the highest median abundance, red $=$ COPD, blue $=$ healthy. Genomes marked with * are those within the discriminatory signature defined for the study cohort (Fig. 2), \# indicates genomes associated with clinical phenotypes (Fig. 3) and ${ }^{\wedge}$ indicates genomes within the disease-associated network (Fig. 6). COPD: $n=16$; healthy: $n=22$.

metagenome functional capacity. These data do, however, validate the association of specific members of the gut microbiome with COPD, providing further impetus for their testing in disease models.

\section{Discussion}

We present the first analysis of the human gut microbiome and metabolome in COPD to complement previous work focused on the lung. We reveal that both the faecal microbiome and metabolome of stable COPD patients are significantly different from that of healthy controls. There was no difference in microbiome composition between current smokers compared to non-smokers with COPD, supporting this as a disease-associated phenotype rather than one driven by the influence of cigarette smoke on the gut microbiome ${ }^{59}$. Several elements of the newly described COPD gut metabolome suggest altered systemic metabolism associated with the disease, the outcomes of which are detectable in faecal samples promoting faecal sampling as a means of monitoring disease. Since changes in metagenomes correlated with disease features, the processes involved may have the potential to be therapeutic targets or the outputs used as faecal biomarkers, although this would need clinical and experimental validation.

We found increased abundance of several Streptococcus species, including S. parasanguinis_B and S. salivarius in COPD, which was partially replicated in an independent validation cohort. Streptococcus enrichment was associated with increased abundance of glucosyltransferase and LPXTG-anchored adhesion domains, suggesting that adhesive capacity was key to increased abundance. Streptococci are pioneer colonisers and some of the first species detected in the oral cavity and gut of infants ${ }^{28}$. Increased abundance of Streptococcus in the gut has been observed in association with smoking ${ }^{60}$, and several studies of the lung microbiome of COPD patients have also noted an increased abundance of the genus ${ }^{13,14}$. S. parasanguinis_B was also isolated from the sputum of a COPD patient experiencing an acute exacerbation (GCF_000963275.16) ${ }^{61}$. One possible explanation for the presence of these organisms in both the lung and gut is a transfer from the oral microbiome. Streptococcus strains exhibit frequent oral-faecal transmission in healthy adults ${ }^{62}$, and transmission rates may increase in COPD where microaspiration of the airways with pharyngeal secretions is exaggerated ${ }^{63}$. Increased Streptococcus across distinct mucosal niches in addition to the non-uniform progression to COPD amongst smokers ${ }^{6}$, also supports a potential genetic predisposition associated with this phenomenon, such as altered mucosal immunity ${ }^{64}$ or antibody secretion $^{65}$, although twin-based analysis suggests environment rather than genotype as the primary explanatory variable in oral streptococci abundance ${ }^{66}$.

While streptococci were associated with COPD status, we found a limited correlation between Streptococcus species abundance and lung function and no correlation with other disease metrics. Multiple members of the family Lachnospiraceae were correlated with reduced lung function. Lachnospiraceae members have been associated with both healthy ${ }^{67,68}$ and diseaseassociated ${ }^{69,70}$ gut microbiomes, and a subset of Dorea species has also been associated with the release of inflammatory cytokines $^{71}$. Contrasting phenotypic effects within genera highlight the interspecies variability that complicates microbiome data interpretation and prevents extrapolation to uncharacterised species such as those described here. Further work is required to determine whether the identified species are actively contributing to the established relationship between airway neutrophilia and lung function decline in $\mathrm{COPD}^{72}$, or whether they are responding to altered conditions independently associated with the disease.

Two metabolites reduced in COPD patients are cofactors of carbamoyl phosphate synthetase I, the first enzyme in the urea cycle, the native cofactor, $\mathrm{N}$-acetylglutamate and its structural analogue, N-carbamoylglutamate. N-carbamoylglutamate has been characterised in the livestock industry due to its capacity to stimulate arginine synthesis ${ }^{53}$. Arginine is an important mediator of gut health ${ }^{73}$ and also contributes to airway function ${ }^{74}$. We found no difference in the concentration of arginine (or other 
urea cycle intermediates) between COPD and healthy individuals. However, analysis of BALF from patients identified a negative association between several amino acids, including arginine and lung function ${ }^{75}$, suggesting that there may be a systemic effect of reduced cofactor levels that does not appear in the faeces. Ncarbamoylglutamate has also been associated with omega-3 fatty acid intake in humans, and a possible link between bacterial production of N-carbamoylglutamate and fatty acids has been suggested $^{76}$; however, it is currently unknown which bacteria may be producing the compound.

We also observed reduced levels of dicarboxylic acids in COPD patients, potentially driven by increased statin use within the cohort. These metabolites are generated endogenously via the omega-oxidation of fatty acids and are excreted in the urine, with increased levels associated with a number of diseases ${ }^{77}$. Two of the dicarboxylic acids identified as depleted, suberate (C8) and sebacate (C10), along with azelate (C9), were identified as positively associated with FEV based on serum analysis, however, were not significantly associated with a diagnosis of $\mathrm{COPD}^{78}$. Statin use was not reported in that study. Impaired fatty acid metabolism has been indicated in COPD based on reduced fatty acid oxidation by isolated peripheral blood mononuclear cells from patients compared to those from healthy smokers ${ }^{79}$. Reduced levels of $\beta$-oxidation in female, but not male, COPD patients are also suggested based on serum analysis ${ }^{80}$. A shift in lipid metabolism may therefore still be associated with COPD; however, it may require a larger cohort to tease apart from the influence of medication. A decrease in dicarboxylic acids has also been observed in association with inflammatory bowel disease $^{81,82}$; however, it is possible that medication profiles also affect these outcomes.

Interestingly, we observed a lower dietary fibre intake in participants with COPD compared to controls based on dietary surveys, which may contribute to both differences in gut microbiome profile and COPD pathology. Dietary fibre resists digestion in the small intestine and upon reaching the colon, soluble forms are partially fermented by commensal bacteria. Some soluble fibres act as prebiotics, providing a selective growth substrate, leading to changes in bacterial number and diversity and increased production of immunosuppressive by-products ${ }^{21}$, which have been shown to reduce airway inflammation in both animal ${ }^{83}$ and humans ${ }^{84,85}$ models of asthma. Hence, increasing fibre intake in COPD may be a relevant therapeutic strategy, as previously suggested ${ }^{26}$.

Analysis and integration of omic datasets are challenging due to the many variables that can influence associations, resulting in a suboptimal rate of validation in the laboratory ${ }^{82}$. Here we attempt to confirm observed microbe-metabolite associations using the encoded genetic potential of the species in question, focusing on species and compounds identified as distinct between COPD and healthy samples. Although we observed overlap in genetic potential, we did not find a clear connection between the datasets. While this may be due to the action of external factors, notably medication, it is also possible that the species responsible for the metabolite signature are not differentially abundant between groups. Rather, differential activity levels, triggered by disease-specific environmental variables and uncaptured by inferred metabolic potential, may induce the signature. To assess this, complementary meta-transcriptomic or proteomic analyses of the microbiome are needed and may yield improved integration of microbial and metabolomic datasets.

Recognised variation in gut microbiome profiles between individuals and confounders such as medication status likely limited our ability to detect additional significant taxonomic and functional biomarkers for COPD. However, encouragingly there was a significant overlap between our relatively small study and validation cohorts. Analysis of larger COPD cohorts will likely identify additional significant correlated biomarkers. Our study was also limited to steady-state disease and therefore did not capture the gut environment during disease exacerbation. Longitudinal analysis during exacerbation and recovery would be particularly interesting if paired with a similar sampling of the lung environment to evaluate potential seeding from the gut. A design incorporating such repeated sampling of the same individual would also help overcome the problem of inter-individual variation.

Despite these limitations, a discriminatory signal is present in both the metagenomic and metabolomic datasets supporting the gut as a potential source of disease biomarkers in COPD. These candidates should be further evaluated for their mechanistic and causal involvement in COPD using established animal models $^{7,86,87}$.

\section{Methods}

Patient characteristics. Twenty-eight COPD patients and 29 healthy controls were recruited from John Hunter Hospital, Belmont District Hospital, Newcastle Community Health Centre, Westlakes Community Health centre and Hunter Medical Research Institute (Newcastle, Australia). All participants provided written informed consent, and ethics approval was obtained from the Human Ethics Research Committees of the Hunter New England Local Health District (14/08/20/ 3.02 ) and the University of Newcastle (H-2015-0006). COPD was defined by the GOLD standard of post-bronchodilator $\mathrm{FEV}_{1}<80 \%$ predicted and $\mathrm{FEV}_{1} / \mathrm{FVC}<$ 0.7 , and by physician diagnosis; all were $>40$ years old and had a previous history of smoking. Healthy controls were adults $>40$ years old with no history of cardiac or respiratory disease, and with normal lung function measured by spirometry $\left(\mathrm{FEV}_{1} /\right.$ FVC ratio $>0.7$ and FEV $1>80 \%$ predicted). Participants were excluded if they had received treatment with an antibiotic or oral prednisone, experienced significant abdominal pain, bloating, diarrhoea or respiratory tract infection in the previous 4 weeks, or had a previous history of gastrointestinal disease. Current and exsmokers were not excluded.

For the validation cohort, 16 COPD patients and 22 healthy participants were recruited through the thoracic outpatient clinic at The Prince Charles Hospital and the general population, respectively. All participants provided written informed consent, and ethics approval was obtained from The Prince Charles Hospital Human Research Ethics Committee (HREC/18/QPCH/234) and the University of Queensland (2108001673/HREC/18/QPCH/234). Patients were included in the study if they had COPD as defined by the GOLD guidelines (chronic airflow limitation that is not fully reversible, with post-bronchodilator FEV1/FVC $<70 \%$ and FEV $1<80 \%$ predicted). COPD patients were former smokers of $\geq 10$ years, who are recruited during stability ( $>4$ weeks since an exacerbation). Healthy controls were adults $>40$ years old with no history of cardiac or respiratory disease. Participants were excluded from the study due to any antibiotic or oral corticosteroid use in the past 4 weeks, a current smoker, had comorbid lung disease (e.g. asthma, lung cancer, interstitial lung disease and bronchiectasis) that interferes with the study outcomes, had other co-morbidities with established altered microbiome (including IBD, irritable bowel syndrome), or extreme dietary habits that may significantly impact gut microbiome composition.

Statistical comparison of metadata characteristics between COPD and healthy groups (Supplementary Data 1 and 27) was undertaken in R using either Student's $t$ test (two-sided) or Wilcoxon rank-sum test dependent on normality estimation using Shapiro-Wilk test. Pearson's chi-squared test was used for categorical variables. Comparison of dietary questionnaire responses was undertaken using a Wilcoxon rank-sum test with Benjamini-Hochberg adjustment for multiple comparisons.

Specimen collection. Individuals who consented to participate were first screened via phone interview, and suitable candidates attended the Hunter Medical Research Institute for a formal assessment. Individual history was recorded, including symptoms, medical and medication history, smoking history and completion of a Dietary Questionnaire for Epidemiological Studies (Version 2, Cancer Council Victoria, Australia). For COPD patients, a history of exacerbations in the last 12 months was also recorded and health status measured using the COPD assessment tool. Spirometry (Easyone) was performed post bronchodilator to assess airway obstruction and a plasma sample collected and stored at $-80^{\circ} \mathrm{C}$ Participants were supplied with a faecal collection kit and instructed to collect faeces within $48 \mathrm{~h}$ of their visit. Faecal samples were stored in the participants' freezer until returned frozen for analysis. Samples were stored at $-80^{\circ} \mathrm{C}$ until processed.

DNA extraction and sequencing. DNA was extracted from $\sim 100 \mathrm{mg}$ of faecal material using an initial bead-beating step followed by extraction using a Maxwell 16 Research Instrument (Promega, USA) according to the manufacturer's protocol 
with the Maxwell 16 Tissue DNA Kit (Promega, USA). DNA concentration was measured using a Qubit assay (Life Technologies, USA) and was adjusted to a concentration of $5 \mathrm{ng} / \mu \mathrm{l}$. The $16 \mathrm{~S}$ rRNA gene encompassing the $\mathrm{V} 6-\mathrm{V} 8$ regions were targeted using the $803 \mathrm{~F}\left(5^{\prime}\right.$-TTAGAKACCCBNGTAGTC- $\left.3^{\prime}\right)$ and $1392 \mathrm{R}$ (5'-ACGGGCGGTGWGTRC-3') primers modified to contain Illumina specific adaptor sequences (803F:5'TCGTCGGCAGCGTCAGATGTGTATAAGAGACAG TTAGAKACCCBNGTAGTC3' and 1392wR:5'GTCTCGTGGGCTCGGGTC TCGTGGGCTCGGAGATGTGTATAAGAGACAGACGGGCGGTGWGTRC3'). Library preparation was performed as described, using the workflow outlined by Illumina (\#15044223 Rev.B). In the first stage, PCR products of $590 \mathrm{bp}$ were amplified according to the specified workflow with an alteration in polymerase used to substitute Q5 Hot Start High-Fidelity 2X Master Mix (New England Biolabs, USA) in standard PCR conditions. The resulting PCR amplicons were purified using Agencourt AMPure XP beads (Beckman Coulter, USA). Purified DNA was indexed with unique 8-bp barcodes using Illumina Nextera XT 384 sample Index Kits A-D (\#FC-131-1002, Illumina, USA). Indexed amplicons were pooled in equimolar concentrations and sequenced on the MiSeq Sequencing System (Illumina, USA) using paired-end sequencing with V3 $300 \mathrm{bp}$ according to the manufacturer's protocol. Metagenomic sequencing was performed using the same DNA extractions. Library preparation was performed using the Nextera DNA Library Preparation Kit (Illumina, USA). Libraries were sequenced using the Illumina NextSeq500 platform generating approximately 2 Gbp of 150-bp pairedend reads per sample. Metagenomic sequencing of the validation cohort was undertaken by Microba (Brisbane, Australia) generating approximately $6 \mathrm{Gbp}$ of 150-bp paired-end reads per sample.

16S rRNA gene sequencing analysis. Reads were cleaned of adaptor sequences using Cutadapt v1.1 $1^{88}$ and trimmed using Trimmomatic v0.36 employing a sliding window of 4 bases with an average base quality above 15, followed by hard trimming to 250 bases with the exclusion of reads less than this length. Read statistics are provided in Supplementary Data 28. The remaining forward reads were processed following the QIIME2 workflow ${ }^{90}$ using DADA2 $\mathrm{v}^{1.12^{91}}$ to denoise sequences. Taxonomy assignment was performed on amplicon sequence variants using BLAST v2.8.192 against the SILVA ${ }^{93}$ reference database version 132. Read counts were normalised prior to PCA and heatmap visualisation using logtransformed cumulative-sum scaling implemented within metagenomeSeq v1.24.194. PCA was performed using the rda function and PERMANOVA using the adonis function within the vegan v2.5-5R package ${ }^{95}$. Heatmaps were generated using the heatmap v1.0.12R package ${ }^{96}$. Alpha-diversity was calculated using QIIME v1.8. $0^{90}$ with raw, unfiltered counts. sPLS-DA analysis was conducted using the R package mixOmics v6.6. $2^{31}$ using log-transformed cumulative-sum-scaled values with $10 \times 10$-fold cross-validation, including sequence variants present at $\geq 0.05 \%$ relative abundance in $\geq 3$ samples.

\section{Metagenomic sequence processing and recovery of MAGs. Contaminating} human reads were identified by mapping against the human genome (Homo sapiens.GRCh38, https://www.ncbi.nlm.nih.gov/assembly/2334371) using BWA $\mathrm{v} 0.7 .12^{97}$ requiring a minimum alignment length of 30 bases and maximum of 15 clipped bases for reads to be considered of human origin. Adaptor removal and read trimming were performed using Trimmomatic v0.36 $6^{89}$ with the following settings: LEADING:3 TRAILING:3 SLIDINGWINDOW:4:15 MINLEN:50. Read statistics are provided in Supplementary Data 29. Each sample was assembled independently using Spades v3.12.098 with the -meta flag. Reads were mapped to each resulting assembly using BamM v1.7.3 (https:/github.com/ecogenomics/ BamM) and bins produced using Metabat v2.12.199 with a minimum contig length of 1500 bases. Contamination and completeness of bins from all samples were assessed using CheckM v1.0.11 ${ }^{100}$. Bins with completeness $>80 \%$ and contamination $<7 \%$ were retained and de-replicated using dRep v2.05 ${ }^{101}$ with default settings ( $99 \%$ identity), skipping quality filtering. The taxonomic affiliation of recovered MAGs was determined using the Genome Taxonomy Database (GTDB) Releases 03-RS86 and 04-R89 ${ }^{102}$ using GTDB-Tk v0.3.0 ${ }^{103}$ (Supplementary Data 30).

Metagenomic community profiling. Reads for each sample were mapped to a dereplicated set of 23,936 genomes from NCBI (GTDB Release 03-RS86) ${ }^{102}$ using BamM with minimum seed length of 25 . Genomes with $>1 \times$ coverage or $>1 \%$ of the genome, as determined using Mosdepth v0.2.3 ${ }^{104}$, were retained $(n=1229$, Source Data) and combined with study MAGs for assessment of community composition. dRep ${ }^{101}$ was used to identify overlap ( $99 \%$ identity) between study MAGs and NCBI genomes, where overlap occurred, MAG was retained. Read counts for the final genome set were determined for each sample via mapping using BamM with minimum seed length of 25 bases and subsequent filtering for minimum mapping percentage identity of $95 \%$. Per-genome read counts were scaled to account for genome size whilst maintaining the raw unmapped read percentage for each sample as a reflection of unrepresented diversity. Relative abundance was calculated using scaled read counts as a fraction of total non-host reads per sample. Alpha-diversity was calculated using QIIME v1.8. $0^{90}$ with counts normalised using the size-factor method implemented within the R package DESeq2 v1.22.230.

PCA was conducted using the R package vegan v2.5-195 on data normalised using log-cumulative-sum scaling (log-CSS) implemented within metagenomeSeq v1.22. $0^{94}$. Differential abundance of bacterial taxa between groups was assessed using the Wald test within DESeq2 v1.20.0 $0^{30}$ based on read counts scaled to account for genome size with the Benjamini-Hochberg adjustment for multiple comparisons. The genome-level analysis was conducted using genomes present with at least $0.05 \%$ relative abundance in one sample. sPLS-DA analysis was conducted using the R package mixOmics v6.6.21 transformed relative abundance with $50 \times 15$-fold cross-validation. Correlation analysis between metagenomic and phenotypic data was undertaken using genomes identified as significantly different between COPD and healthy samples following removal of patient confounders (Supplementary Data 11). Spearman's rho was calculated using 'corr.test' function within R package psych v1.8.12105 based on centred log-ratio-transformed genome relative abundance. A correlation matrix was produced using 'corrplot' function with $\mathrm{R}$ package corrplot $\mathrm{v} 0.84^{106}$.

Metagenomic functional profiling. For read-based analysis, protein fragments in raw reads were predicted using Prodigal v2.6.3 ${ }^{107}$ and subsequently alignment with HMMER v3.1b2 $2^{108}$ to the hidden Markov model databases dbCAN CAZy v6 ${ }^{109}$, Pfam $\mathrm{r} 31^{110}$ and TIGRFAM v15 ${ }^{111}$ with a maximum e-value cut-off of $1 \mathrm{e}-10$. KEGG orthology was determined via BLAST v2.8.192 alignment to UniProt UniRef100 database downloaded on July $2017^{112}$ with maximum e value of $1 \mathrm{e}-10$ and subsequent extraction of associated KO terms. Counts per sample were used to compare group functional profiles with DESeq2 v1.20.0 30 following removal of domains with total read counts $\leq 10 \%$ of the average read count across all domains Genome-level analysis of KEGG orthology terms and module completeness was undertaken using EnrichM v0.5.0 (https://github.com/geronimp/enrichM) with maximum e value of $1 \mathrm{e}-10$ and Fisher's exact test with Benjamini-Hochberg adjustment used to assess significance. Comparison of module completeness was undertaken in $\mathrm{R}$ using the Wilcoxon rank-sum test with Benjamini-Hochberg adjustment. The presence of genes of interest (i.e. related to an enriched metabolite) in enriched genomes was determined using BLAST with minimum e value $1 \mathrm{e}-10$, identity $30 \%$ and alignment length $70 \%$. Protein sequences used as queries are included in Supplementary Data 26.

Metabolite extraction, profiling and analysis. Metabolites were profiled in faecal samples by Metabolon Inc. (Durham, NC, USA). All samples were maintained at $-80^{\circ} \mathrm{C}$ until processed as previously described ${ }^{113}$. Global metabolic profiles were determined using the Metabolon HD4 platform. Samples were prepared using the automated MicroLab STAR ${ }^{\circ}$ system (Hamilton Company, USA), with several recovery standards added prior to extraction and processing for quality control. To recover chemically diverse metabolites and precipitate protein and dissociate small molecules bound to protein in the precipitated matrix, samples were extracted with methanol with vigorous shaking for 2 min (Glen Mills GenoGrinder 2000, USA) followed by centrifugation. The extract was divided into five different fractions for further analysis. The organic solvent was removed by placing briefly on a TurboVap ${ }^{\oplus}$ Concentration Evaporator (Zymark). Samples were stored overnight under nitrogen.

The process of ultra performance liquid chromatography (UPLC)/mass spectrometry (MS)/MS was performed with a Waters ACQUITY (UPLC), Thermo Scientific Q-Exactive high-resolution mass spectrometer interfaced with a heated electrospray ionisation (HESI-II) source and Orbitrap mass analyser operated at 35,000 mass resolution. Sample extracts were processed dry and reconstituted to consist of a series of standards at fixed concentrations to have injection and chromatography consistency before detailed analysis with four methods. For more hydrophilic compounds, optimised reverse-phase UPLC-MS/MS with acidic conditions and positive ion-mode electrospray ionisation was used. Here, a C18 column (Waters UPLC BEH C18-2.1 $\times 100 \mathrm{~mm}, 1.7 \mu \mathrm{m}$ ), consisting of perfluoropentanoic acid $(0.05 \%)$ and formic acid $(0.1 \%)$ was used to gradient-elute the extract using water and methanol. For hydrophobic compounds, extracts were gradient-eluted with the same $\mathrm{C} 18$ column using methanol, acetonitrile, water, perfluoropentanoic acid $(0.05 \%)$ and formic acid $(0.01 \%)$. Higher organic content was maintained during processing. Basic negative-ion conditions using a separate C18 column were used to elute the basic extract with methanol and water, ammonium bicarbonate (6.5 mM, pH 8). Negative-ion-mode electrospray ionisation conditions with hydrophilic interaction chromatography were used with a Waters UPLC BEH Amide $2.1 \times 150-\mathrm{mm}, 1.7-\mu \mathrm{m}$ column. Here, extracts were gradient-eluted with water and acetonitrile with ammonium formate $(10 \mathrm{mM}, \mathrm{pH}$ 10.8). The mass spectrometry analysis alternated between MS and data-dependent $\mathrm{MS}^{\mathrm{n}}$ scans, with scan range covering from (70 to $1000 \mathrm{~m} / \mathrm{z}$ ) achieved with the dynamic elusion method ${ }^{114}$.

Metabolon's hardware and software systems were based on LAN backbone; database servers operating on Oracle 10.2.0.1 Enterprise Edition, are utilised to extract, peak-identify and quality-check and process the raw data files. Compound identification is achieved by comparison with library entries of purified standards (or recurrent unknown entities), which consist of retention time/index, the massto-charge ratio $(\mathrm{m} / \mathrm{z})$ and chromatographic data, including MS/MS spectral data information. Biochemical identification follows the retention time/index window of the proposed identification mass match to the library $( \pm 10 \mathrm{ppm})$ and MS/MS forward and reverse scores. Quality check and curation procedures are followed to ensure that library matches for each compound from each sample are correct. 
Peaks are quantified using area-under-the-curve detector ion counts and corrected across multiple runs by adjusting the median value of each compound to 1 .

Following median scaling, then imputation of missing values, if any, with the minimum observed value for each compound, the data were transformed to the natural $\log$ for statistical analysis. Linear regression of metabolite data was performed using $\mathrm{lm}$ package in R implemented within NormalizeMets ${ }^{115} \mathrm{v} 0.25^{115}$ incorporating sample group, age, BMI and sex and non-COPD medications within the model matrix as indicated.

Metabolomic and metagenomic data integration. Correlation analysis between metagenomic and metabolomic data was undertaken using genomes and metabolites identified as significantly different between COPD and healthy samples incorporating adjustment for age, sex and BMI (Supplementary Data 7 and 21). Spearman's rho was calculated using 'corr.test' function within R package psych v1.8.12 105 based on centred log-ratio- transformed genome relative abundance and log-transformed raw metabolite values. The pseudo count used for each dataset was one order of magnitude below the lowest non-zero value. The correlation matrix was produced using 'corrplot' function with R package corrplot $\mathrm{v} 0.84^{106}$.

DIABLO from the $\mathrm{R}$ package mixOmics v6.6.2 ${ }^{31}$ was used to generate integrated metagenomic and metabolomic signature. The analysis was performed using centred log-ratio-transformed taxa relative abundance (with a pseudo count of $1 \mathrm{e}-08$, one order of magnitude below the lowest non-zero value) and $\log$ transformed median-scaled metabolite data. Taxa were filtered for those present at a minimum of $0.05 \%$ in at least ten samples (genome level) and metabolites for those detected in at least 10 samples. The block link within the design matrix was set at 0.1. The optimum number of components and variables included within the final model was determined using the 'tune.block.splsda' function with $50 \times 10$-fold cross-validation.

Reporting summary. Further information on research design is available in the Nature Research Reporting Summary linked to this article.

\section{Data availability}

The 16S rRNA amplicon and metagenomic sequencing data have been deposited to the NCBI Sequence Read Archive under accession PRJNA562766. Recovered MAGs have been deposited to the NCBI DDBJ/ENA/GenBank database under accessions WGSA00000000-WHIU00000000. Prokka annotated MAG sequences in GenBank format are available at https://github.com/katebowerman/COPD. Sample accessions are provided in Supplementary Data 28-30. Sequence variant read counts from 16 S rRA amplicon sequencing (raw data underlying Fig. 1) and metagenomic genome-based mapping counts (raw data underlying Figs. 2-7) are provided as a Source Data File. The reference human genome used in this study (Homo_sapiens.GRCh38) is available at https://www.ncbi.nlm.nih.gov/assembly/2334371. Reference bacterial genomes are available from https://www.ncbi.nlm.nih.gov/assembly/. Additional databases used in this study are available as follows: SILVA v132, GTDB 03-RS86 and 04-R89, dbCAN v6, Pfam r31, TIGRFAM v15 and UniProt UniRef100. Source data are provided with this paper.

Received: 21 December 2019; Accepted: 19 October 2020; Published online: 18 November 2020

\section{References}

1. Rabe, K. F. \& Watz, H. Chronic obstructive pulmonary disease. Lancet 389, 1931-1940 (2017).

2. Keely, S., Talley, N. J. \& Hansbro, P. M. Pulmonary-intestinal cross-talk in mucosal inflammatory disease. Mucosal Immunol. 5, 7-18 (2012).

3. Naghavi, M. et al. Global, regional, and national age-sex specific mortality for 264 causes of death, 1980-2016: a systematic analysis for the Global Burden of Disease Study 2016. Lancet 390, 1151-1210 (2017).

4. Mannino, D. M. \& Buist, A. S. Global burden of COPD: risk factors, prevalence, and future trends. Lancet 370, 765-773 (2007).

5. Fricker, M. et al. Chronic cigarette smoke exposure induces systemic hypoxia that drives intestinal dysfunction. JCI Insight 3, e94040 (2018).

6. Løkke, A., Lange, P., Scharling, H., Fabricius, P. \& Vestbo, J. Developing COPD: a 25 year follow up study of the general population. Thorax $\mathbf{6 1}$ 935-939 (2006).

7. Jones, B. et al. Animal models of COPD: what do they tell us? Respirology 22, 21-32 (2017)

8. Yang I. A., Clarke M. S., Sim E. H. A., Fong K. M. Inhaled corticosteroids for stable chronic obstructive pulmonary disease. Cochrane Database Syst. Rev. (2012).

9. Calverley, P. M. A. et al. Salmeterol and fluticasone propionate and survival in chronic obstructive pulmonary disease. N. Engl. J. Med. 356, 775-789 (2007).
10. Wedzicha, J. A. \& Seemungal, T. A. R. COPD exacerbations: defining their cause and prevention. Lancet 370, 786-796 (2007).

11. Leung, J. M. et al. The role of acute and chronic respiratory colonization and infections in the pathogenesis of COPD. Respirology 22, 634-650 (2017).

12. Wilkinson, T. M. A. et al. A prospective, observational cohort study of the seasonal dynamics of airway pathogens in the aetiology of exacerbations in COPD. Thorax 72, 919-927 (2017).

13. Wang, Z. et al. Airway host-microbiome interactions in chronic obstructive pulmonary disease. Respir. Res. 20, 113 (2019).

14. Pragman, A. A., Kim, H. B., Reilly, C. S., Wendt, C. \& Isaacson, R. E. The lung microbiome in moderate and severe chronic obstructive pulmonary disease. PLoS ONE 7, e47305 (2012).

15. Ren, L. et al. Transcriptionally active lung microbiome and its association with bacterial biomass and host inflammatory status. mSystems 3, e00199-00118 (2018).

16. Sze, M. A. et al. Host response to the lung microbiome in chronic obstructive pulmonary disease. Am. J. Respir. Crit. Care Med. 192, 438-445 (2015).

17. Huffnagle, G. B., Dickson, R. P. \& Lukacs, N. W. The respiratory tract microbiome and lung inflammation: a two-way street. Mucosal Immunol. 10, 299 (2016).

18. Budden, K. F. et al. Functional effects of the microbiota in chronic respiratory disease. Lancet Respir. Med. 7, 907-920 (2019).

19. Budden, K. F. et al. Emerging pathogenic links between microbiota and the gut-lung axis. Nat. Rev. Microbiol. 15, 55 (2016).

20. Arrieta, M. C. et al. Early infancy microbial and metabolic alterations affect risk of childhood asthma. Sci. Transl. Med. 7, 307ra152 (2015).

21. Trompette, A. et al. Gut microbiota metabolism of dietary fiber influences allergic airway disease and hematopoiesis. Nat. Med. 20, 159 (2014).

22. Thorburn, A. N. et al. Evidence that asthma is a developmental origin disease influenced by maternal diet and bacterial metabolites. Nat. Commun. 6, 7320 (2015).

23. Trompette, A. et al. Dietary fiber confers protection against flu by shaping $\mathrm{Ly}_{6 \mathrm{c}^{-}}$patrolling monocyte hematopoiesis and $\mathrm{CD}^{+} \mathrm{T}$ cell metabolism. Immunity 48, 992-1005.e1008 (2018).

24. Ekbom, A., Brandt, L., Granath, F., Löfdahl, C.-G. \& Egesten, A. Increased risk of both ulcerative colitis and Crohn's disease in a population suffering from COPD. Lung 186, 167-172 (2008).

25. Mateer, S. W. et al. Potential mechanisms regulating pulmonary pathology in inflammatory bowel disease. J. Leukoc. Biol. 98, 727-737 (2015).

26. Vaughan, A., Frazer, Z. A., Hansbro, P. M. \& Yang, I. A. COPD and the gutlung axis: the therapeutic potential of fibre. J. Thorac. Dis. 11, S2173-S2180 (2019).

27. Chunxi, L., Haiyue, L., Yanxia, L., Jianbing, P. \& Jin, S. The gut microbiota and respiratory diseases: new evidence. J. Immunol. Res 2020, 2340670-2340670 (2020).

28. Ferretti, P. et al. Mother-to-infant microbial transmission from different body sites shapes the developing infant gut microbiome. Cell Host Microbe 24, 133-145.e135 (2018).

29. Franzosa, E. A. et al. Identifying personal microbiomes using metagenomic codes. Proc. Natl Acad. Sci. USA 112, E2930-E2938 (2015).

30. Love, M. I., Huber, W. \& Anders, S. Moderated estimation of fold change and dispersion for RNA-seq data with DESeq2. Genome Biol. 15, 550 (2014).

31. Rohart, F., Gautier, B., Singh, A., Lê \& Cao, K.-A. mixOmics: an R package for 'omics feature selection and multiple data integration. PLoS Comput. Biol. 13 e1005752 (2017).

32. McCabe, R. M. \& Donkersloot, J. A. Adherence of Veillonella species mediated by extracellular glucosyltransferase from Streptococcus salivarius. Infect. Immun. 18, 726-734 (1977).

33. McNab, R. et al. Cell wall-anchored CshA polypeptide (259 kilodaltons) in Streptococcus gordonii forms surface fibrils that confer hydrophobic and adhesive properties. J. Bacteriol. 181, 3087-3095 (1999).

34. Kolachala, V. L. et al. Epithelial-derived fibronectin expression, signaling, and function in intestinal inflammation. J. Biol. Chem. 282, 32965-32973 (2007).

35. Dammeier, J., Brauchle, M., Falk, W., Grotendorst, G. R. \& Werner, S. Connective tissue growth factor: a novel regulator of mucosal repair and fibrosis in inflammatory bowel disease? Int J. Biochem. Cell Biol. 30, 909-922 (1998).

36. Annoni, R. et al. Extracellular matrix composition in COPD. Eur. Respir. J. 40, 1362-1373 (2012).

37. Liu, G. et al. Fibulin-1 regulates the pathogenesis of tissue remodeling in respiratory diseases. JCI Insight 1, e86380 (2016).

38. Bensing, B. A., Seepersaud, R., Yen, Y. T. \& Sullam, P. M. Selective transport by SecA2: an expanding family of customized motor proteins. Biochim. Biophys. Acta 1843, 1674-1686 (2014).

39. Eijkelkamp, B. A., McDevitt, C. A. \& Kitten, T. Manganese uptake and streptococcal virulence. Biometals 28, 491-508 (2015).

40. Nayfach, S., Shi, Z. J., Seshadri, R., Pollard, K. S. \& Kyrpides, N. C. New insights from uncultivated genomes of the global human gut microbiome. Nature 568, 505-510 (2019). 
41. Mingrone, G., Castagneto-Gissey, L. \& Macé, K. Use of dicarboxylic acids in type 2 diabetes. Br. J. Clin. Pharm. 75, 671-676 (2013).

42. Miura, Y. The biological significance of $\omega$-oxidation of fatty acids. Proc. Jpn Acad. Ser. B Phys. Biol. Sci. 89, 370-382 (2013).

43. Parke, D., Garcia, M. A. \& Ornston, L. N. Cloning and genetic characterization of $d c a$ genes required for $\beta$-oxidation of straight-chain dicarboxylic acids in Acinetobacter sp. strain ADP1. Appl. Environ. Microbiol. 67, 4817-4827 (2001).

44. Park, H. S. et al. Statins increase mitochondrial and peroxisomal fatty acid oxidation in the liver and prevent non-alcoholic steatohepatitis in mice. Diabetes Metab. J. 40, 376-385 (2016).

45. Jacobs, J. P. et al. A disease-associated microbial and metabolomics state in relatives of pediatric inflammatory bowel disease patients. Cell. Mol. Gastroenterol. Hepatol. 2, 750-766 (2016).

46. Ma, W. et al. Advances in cadaverine bacterial production and its applications. Engineering 3, 308-317 (2017).

47. Shi, X., Yao, D. \& Chen, C. Identification of $\mathrm{N}$-acetyltaurine as a novel metabolite of ethanol through metabolomics-guided biochemical analysis. $J$. Biol. Chem. 287, 6336-6349 (2012).

48. Landa, M., Burns, A. S., Roth, S. J. \& Moran, M. A. Bacterial transcriptome remodeling during sequential co-culture with a marine dinoflagellate and diatom. ISME J. 11, 2677-2690 (2017).

49. Hession, A. O., Esrey, E. G., Croes, R. A. \& Maxwell, C. A. N-Acetylglutamate and N-Acetylaspartate in soybeans (Glycine max L.), maize (Zea maize L.), and other foodstuffs. J. Agric. Food Chem. 56, 9121-9126 (2008).

50. Caldovic, L. \& Tuchman, M. N-acetylglutamate and its changing role through evolution. Biochem. J. 372, 279-290 (2003).

51. Sankaranarayanan, K. et al. Gut microbiome diversity among Cheyenne and Arapaho individuals from western Oklahoma. Curr. Biol. 25, 3161-3169 (2015).

52. Henriksen, C. M., Nielsen, J. \& Villadsen, J. Cyclization of alpha-aminoadipic acid into the delta-lactam 6-oxo-piperidine-2-carboxylic acid by Penicillium chrysogenum. J. Antibiot. 51, 99-106 (1998).

53. Wu, G., Knabe, D. A. \& Kim, S. W. Arginine nutrition in neonatal pigs. J. Nutr. 134, 2783S-2790S (2004)

54. Cao, W. et al. Dietary arginine and N-carbamylglutamate supplementation enhances the antioxidant statuses of the liver and plasma against oxidative stress in rats. Food Funct. 7, 2303-2311 (2016).

55. Wu, X., Zhang, Y., Liu, Z., Li, T. J. \& Yin, Y. L. Effects of oral supplementation with glutamate or combination of glutamate and $\mathrm{N}$-carbamylglutamate on intestinal mucosa morphology and epithelium cell proliferation in weanling piglets. J. Anim. Sci. 90, 337-339 (2012).

56. Kodani, S., Imoto, A., Mitsutani, A. \& Murakami, M. Isolation and identification of the antialgal compound, harmane (1-methyl- $\beta$-carboline), produced by the algicidal bacterium, Pseudomonas sp. K44-1. J. Appl. Phycol. 14, 109-114 (2002).

57. Arshad, N., Zitterl-Eglseer, K., Hasnain, S. \& Hess, M. Effect of Peganum harmala or its $\beta$-carboline alkaloids on certain antibiotic resistant strains of bacteria and protozoa from poultry. Phytother. Res. 22, 1533-1538 (2008).

58. Jakobsen, H. et al. The alkaloid compound harmane increases the lifespan of Caenorhabditis elegans during bacterial infection, by modulating the nematode's innate immune response. PLoS ONE 8, e60519 (2013).

59. Biedermann, L. et al. Smoking cessation induces profound changes in the composition of the intestinal microbiota in humans. PLoS ONE 8, e59260 (2013).

60. Shanahan, E. R. et al. Influence of cigarette smoking on the human duodenal mucosa-associated microbiota. Microbiome 6, 150 (2018).

61. Chan, K.-G. et al. Genome anatomy of Streptococcus parasanguinis strain C1A, isolated from a patient with acute exacerbation of chronic obstructive pulmonary disease, reveals unusual genomic features. Genome Announc. 3, e00541-00515 (2015).

62. Schmidt, T. S. et al. Extensive transmission of microbes along the gastrointestinal tract. eLife 8, e42693 (2019).

63. Cvejic, L. et al. Laryngeal penetration and aspiration in individuals with stable COPD. Respirology 16, 269-275 (2011).

64. Igartua, C. et al. Host genetic variation in mucosal immunity pathways influences the upper airway microbiome. Microbiome 5, 16 (2017).

65. Wacklin, P. et al. Secretor genotype (FUT2 gene) is strongly associated with the composition of bifidobacteria in the human intestine. PLoS ONE 6, e20113 (2011).

66. Gomez, A. et al. Host genetic control of the oral microbiome in health and disease. Cell Host Microbe 22, 269-278.e263 (2017).

67. Zhang, J. et al. A phylo-functional core of gut microbiota in healthy young Chinese cohorts across lifestyles, geography and ethnicities. ISME J. 9, 1979-1990 (2015).

68. Sekelja, M., Berget, I., Næs, T. \& Rudi, K. Unveiling an abundant core microbiota in the human adult colon by a phylogroup-independent searching approach. ISME J. 5, 519-531 (2011).
69. Zuo, K. et al. Disordered gut microbiota and alterations in metabolic patterns are associated with atrial fibrillation. Gigascience 8, giz058 (2019).

70. Gomes, A. C., Hoffmann, C. \& Mota, J. F. The human gut microbiota: metabolism and perspective in obesity. Gut Microbes 9, 308-325 (2018).

71. Schirmer, M. et al. Linking the human gut microbiome to inflammatory cytokine production capacity. Cell 167, 1125-1136.e1128 (2016).

72. Hoenderdos, K. \& Condliffe, A. The neutrophil in chronic obstructive pulmonary disease. Am. J. Respir. Cell Mol. Biol. 48, 531-539 (2013).

73. Fritz, J. H. Arginine cools the inflamed gut. Infect. Immun. 81, 3500-3502 (2013).

74. Maarsingh, H., Zaagsma, J. \& Meurs, H. Arginine homeostasis in allergic asthma. Eur. J. Pharm. 585, 375-384 (2008).

75. Halper-Stromberg, E. et al. Bronchoalveolar lavage fluid from COPD patients reveals more compounds associated with disease than matched plasma. Metabolites 9, 157 (2019).

76. Menni, C. et al. Omega-3 fatty acids correlate with gut microbiome diversity and production of $\mathrm{N}$-carbamylglutamate in middle aged and elderly women. Sci. Rep. 7, 11079 (2017).

77. Wanders, R. J. A., Komen, J. \& Kemp, S. Fatty acid omega-oxidation as a rescue pathway for fatty acid oxidation disorders in humans. FEBS J. 278, 182-194 (2011).

78. Yu, B. et al. Metabolomics identifies novel blood biomarkers of pulmonary function and COPD in the general population. Metabolites 9, 61 (2019).

79. Agarwal, A. R. et al. Systemic immuno-metabolic alterations in chronic obstructive pulmonary disease (COPD). Respir. Res. 20, 171 (2019).

80. Naz, S. et al. Metabolomics analysis identifies sex-associated metabotypes of oxidative stress and the autotaxin-lysoPA axis in COPD. Eur. Respir. J. 49, 1602322 (2017).

81. Lee, T. et al. Oral versus intravenous iron replacement therapy distinctly alters the gut microbiota and metabolome in patients with IBD. Gut 66, 863-871 (2017).

82. Franzosa, E. A. et al. Gut microbiome structure and metabolic activity in inflammatory bowel disease. Nat. Microbiol. 4, 293-305 (2019).

83. Maslowski, K. M. et al. Regulation of inflammatory responses by gut microbiota and chemoattractant receptor GPR43. Nature 461, 1282-1286 (2009).

84. McLoughlin, R. et al. Soluble fibre supplementation with and without a probiotic in adults with asthma: a 7-day randomised, double blind, three way cross-over trial. EBioMedicine 46, 473-485 (2019).

85. Halnes, I. et al. Soluble fibre meal challenge reduces airway inflammation and expression of GPR43 and GPR41 in asthma. Nutrients 9, 57 (2017).

86. Beckett, E. L. et al. A new short-term mouse model of chronic obstructive pulmonary disease identifies a role for mast cell tryptase in pathogenesis. $J$. Allergy Clin. Immunol. 131, 752-762 (2013).

87. Hansbro, P. M. et al. Importance of mast cell Prss31/transmembrane tryptase/ tryptase-gamma in lung function and experimental chronic obstructive pulmonary disease and colitis. J. Biol. Chem. 289, 18214-18227 (2014).

88. Martin, M. Cutadapt removes adapter sequences from high-throughput sequencing reads. EMBnetjournal 17, 10-12 (2011).

89. Bolger, A. M., Lohse, M. \& Usadel, B. Trimmomatic: a flexible trimmer for Illumina sequence data. Bioinformatics 30, 2114-2120 (2014).

90. Caporaso, J. G. et al. QIIME allows analysis of high-throughput community sequencing data. Nat. Methods 7, 335-336 (2010).

91. Callahan, B. J. et al. DADA2: High-resolution sample inference from Illumina amplicon data. Nat. Methods 13, 581 (2016).

92. Altschul, S. F., Gish, W., Miller, W., Myers, E. W. \& Lipman, D. J. Basic local alignment search tool. J. Mol. Biol. 215, 403-410 (1990).

93. Quast, C. et al. The SILVA ribosomal RNA gene database project: improved data processing and web-based tools. Nucleic Acids Res. 41, D590-D596 (2013).

94. Paulson, J. N., Stine, O. C., Bravo, H. C. \& Pop, M. Differential abundance analysis for microbial marker-gene surveys. Nat. Methods 10, 1200-1202 (2013).

95. Oksanen J. et al. vegan: Community Ecology Package. R Package Version 231. http://CRAN.R-project.org/package $=$ vegan (2015).

96. Kolde R. pheatmap: Pretty Heatmaps. R Package Version 107. http://CRAN.Rproject.org/package $=$ pheatmap (2015).

97. Li, H. \& Durbin, R. Fast and accurate short read alignment with BurrowsWheeler transform. Bioinformatics 25, 1754-1760 (2009).

98. Nurk, S., Meleshko, D., Korobeynikov, A. \& Pevzner, P. A. metaSPAdes: a new versatile metagenomic assembler. Genome Res 27, 824-834 (2017).

99. Kang, D. D., Froula, J., Egan, R. \& Wang, Z. MetaBAT, an efficient tool for accurately reconstructing single genomes from complex microbial communities. PeerJ 3, e1165 (2015).

100. Parks, D. H., Imelfort, M., Skennerton, C. T., Hugenholtz, P. \& Tyson, G. W. CheckM: assessing the quality of microbial genomes recovered from isolates, single cells, and metagenomes. Genome Res. 25, 1043-1055 (2015). 
101. Olm, M. R., Brown, C. T., Brooks, B. \& Banfield, J. F. dRep: a tool for fast and accurate genomic comparisons that enables improved genome recovery from metagenomes through de-replication. ISME J. 11, 2864-2868 (2017).

102. Parks, D. H. et al. A standardized bacterial taxonomy based on genome phylogeny substantially revises the tree of life. Nat. Biotechnol. 36, 996 (2018).

103. Chaumeil, P.-A., Mussig, A. J., Hugenholtz, P. \& Parks, D. H. GTDB-Tk: a toolkit to classify genomes with the Genome Taxonomy Database. Bioinformatics 36, 1925-1927 (2019).

104. Pedersen, B. S. \& Quinlan, A. R. Mosdepth: quick coverage calculation for genomes and exomes. Bioinformatics 34, 867-868 (2018).

105. Revelle W. psych: Procedures for Personality and Psychological Research. R Package Version 1812. https://CRAN.R-project.org/package=psych (2018).

106. Wei T., Simko V. corrplot: Visualization of a Correlation Matrix. R Package Version 084. https://github.com/taiyun/corrplot (2018).

107. Hyatt, D. et al. Prodigal: prokaryotic gene recognition and translation initiation site identification. BMC Bioinform. 11, 119 (2010).

108. Mistry, J., Finn, R. D., Eddy, S. R., Bateman, A. \& Punta, M. Challenges in homology search: HMMER3 and convergent evolution of coiled-coil regions. Nucleic Acids Res. 41, e121 (2013).

109. Yin, Y. et al. dbCAN: a web resource for automated carbohydrate-active enzyme annotation. Nucleic Acids Res. 40, W445-W451 (2012).

110. Finn, R. D. et al. Pfam: the protein families database. Nucleic Acids Res. 42, D222-D230 (2014)

111. Haft, D. H., Selengut, J. D. \& White, O. The TIGRFAMs database of protein families. Nucleic Acids Res. 31, 371-373 (2003).

112. Suzek, B. E., Huang, H. Z., McGarvey, P., Mazumder, R. \& Wu, C. H. UniRef: comprehensive and non-redundant UniProt reference clusters. Bioinformatics 23, 1282-1288 (2007).

113. Evans, A. M. et al. High resolution mass spectrometry improves data quantity and quality as compared to unit mass resolution mass spectrometry in highthroughput profiling metabolomics. Metabolomics 4, 1000132 (2014).

114. DeHaven, C. D., Evans, A. M., Dai, H. \& Lawton, K. A. Organization of GC/ MS and LC/MS metabolomics data into chemical libraries. J. Cheminform. 2, 9 (2010).

115. De Livera, A. M., Olshansky, G., Simpson, J. A. \& Creek, D. J. NormalizeMets: assessing, selecting and implementing statistical methods for normalizing metabolomics data. Metabolomics 14, 54 (2018).

\section{Acknowledgements}

The authors thank Professor Graham Giles of the Cancer Epidemiology Centre of The Cancer Council Victoria, for permission to use the Dietary Questionnaire for Epidemiological Studies (Version 2), Melbourne: The Cancer Council Victoria, 1996. We thank Lorissa Hopkins and Jasmine Wark for assistance with recruiting patients, collection of human data and samples. This work was funded by grants from the Rainbow Foundation to P.M.H., the National Health and Medical Research Council (NHMRC, 1059238) of Australia to P.M.H. and P.H. and Prince Charles Hospital Foundation
Innovations Grants to I.A.Y. (INN2018-30) and A.V. (INN2019-24). P.M.H. is funded by fellowships from the NHMRC (1079187 and 1175134) and A.V. by a fellowship from the Prince Charles Hospital Foundation (RF2017-05).

\section{Author contributions}

Study design (S.L.G., K.F.B., P.A.W., P.H. and P.M.H.), questionnaire selection (S.L.G., K.F.B. and L.G.W.), data collection (S.F.R. and K.F.B.), sample collection (P.A.W., A.V and I.A.Y.), sample processing (N.L., S.L.G., K.F.B., S.F.R. and S.D.S.), data processing (K.L.B., S.F.R. and D.L.A.W.), data analysis (K.L.B. and S.F.R.), paper preparation (K.L.B., S.F.R., L.G.W., P.H. and P.M.H.) and paper editing and review (K.L.B., S.F.R., A.V., N.L., K.F.B., R.Y.K., D.L.A.W., S.D.S., L.G.W., I.A.Y., P.A.W., P.H. and P.H.M.)

\section{Competing interests}

P.H. is a co-founder of Microba Life Sciences Limited, and D.L.A.W. is currently an employee of Microba. The remaining authors declare no competing interests.

\section{Additional information}

Supplementary information is available for this paper at https://doi.org/10.1038/s41467020-19701-0.

Correspondence and requests for materials should be addressed to P.M.H.

Peer review information Nature Communications thanks James Brown and the other, anonymous, reviewer(s) for their contribution to the peer review of this work. Peer reviewer reports are available.

Reprints and permission information is available at http://www.nature.com/reprints

Publisher's note Springer Nature remains neutral with regard to jurisdictional claims in published maps and institutional affiliations.

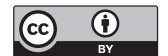

Open Access This article is licensed under a Creative Commons Attribution 4.0 International License, which permits use, sharing, adaptation, distribution and reproduction in any medium or format, as long as you give appropriate credit to the original author(s) and the source, provide a link to the Creative Commons license, and indicate if changes were made. The images or other third party material in this article are included in the article's Creative Commons license, unless indicated otherwise in a credit line to the material. If material is not included in the article's Creative Commons license and your intended use is not permitted by statutory regulation or exceeds the permitted use, you will need to obtain permission directly from the copyright holder. To view a copy of this license, visit http://creativecommons.org/ licenses/by/4.0/.

(C) The Author(s) 2020 